\title{
Emerging X-ray imaging technologies for energy materials
}

Chuntian Cao ${ }^{a, c}$, Michael F. Toneyc, Tsun-Kong Sham ${ }^{d}$, Ross Hardere, Paul R. Shearing ${ }^{f}$, Xianghui Xiaog $^{g}$, Jiajun Wang ${ }^{a, b, h, *}$

a School of Chemistry and Chemical Engineering, Harbin Institute of Technology, Harbin, 150001 China

${ }^{b}$ MIIT Key Laboratory of Critical Materials Technology for New Energy Conversion and Storage, Harbin Institute of Technology, Harbin, 150001, China

c SSRL Materials Science Division, SLAC National Accelerator Laboratory, Menlo Park, California 94025, United States

${ }^{\mathrm{d}}$ Department of Chemistry, University of Western Ontario, London, Ontario N6A 5B9, Canada

e Advanced Photon Source, Argonne National Laboratory, Lemont, Illinois 60439, United States

${ }^{f}$ Department of Chemical Engineering, Uneversity College London, Torrington Place, London WC1E 7JE, United Kingdom

g National Synchrotron Light Source II, Brookhaven National Laboratory, Upton, New York 11973, United States

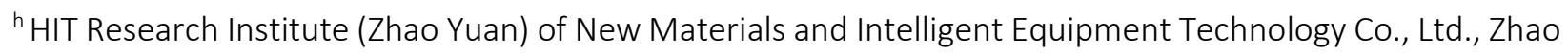
Yuan, 265400, China

* Corresponding author: Wang, J. (jiajunhit@hit.edu.cn)

\section{Abstract}

With the growing need for sustainable energy technologies, advanced characterization methods become more and more critical for optimizing energy materials and understanding their operation mechanisms. In this review, we focus on the synchrotron-based X-ray imaging technologies, and the associated applications in gaining fundamental insights into the physical/chemical properties and reaction mechanisms of energy materials. We will discuss a few major X-ray imaging technologies, including $X$-ray projection imaging, transmission $\mathrm{X}$-ray microscopy, scanning transmission $\mathrm{X}$-ray microscopy, tender and soft $\mathrm{X}$-ray imaging, and coherent diffraction imaging. Researchers can choose from various $X$-ray imaging techniques with different working principles based on research goals and sample specifications. With the X-ray imaging techniques, we can obtain the morphology, phase, lattice and strain information of energy materials in both 2D and 3D in an intuitive way. In addition, due to the high penetration of X-rays, operando/in-situ experiments can be designed to track the qualitative and quantitative changes of the samples during operation. We expect this review can broaden reader's view on X-ray imaging techniques and inspire new ideas and possibilities in energy materials research.

\section{Introduction}


Foreseeing a shortage of traditional energy sources in the future, the world has an increasing demand for renewable energy sources and sustainable energy technologies including but not limited to rechargeable batteries, solar cells, and fuel cells. In addition to a large amount of research on developing new energy materials, effort in characterization methods is indispensable in order to explore the physical and chemical properties of materials, to understand the operation mechanisms of devices, and to ultimately improve the performance of renewable energy systems. Some commonly used characterization methods include electron microscopes, spectroscopies, nuclear magnetic resonance, X-ray scattering techniques, and X-ray imaging techniques. [1-7] In this review, we will focus on how synchrotron-based X-ray imaging techniques are applied in the qualitative and quantitative characterization of energy materials. The advent of third generation synchrotron light sources and beyond and accompanying optics and detector developments have helped propel the advancement of synchrotron microscopy (from infrared to hard X-ray), as it is evident from recent conference proceedings. [8] These techniques are used throughout the synthesis and working processes of energy materials, and can assist in the development of sustainable energy technologies and aid the design and the optimization of next-generation devices.

Imaging techniques have the advantage of giving information in an intuitive way, known as "what you see is what you get". The high-resolution X-ray imaging bridges the gap between optical and electron imaging, and the high penetration capability of $\mathrm{X}$-rays allows for noninvasive characterization and in-situ/operando experiments. Additionally, X-ray imaging is significantly less damaging compared with electron microscopes. $X$-ray imaging techniques have been further developed benefiting from the advancement of synchrotron radiation sources which provide $\mathrm{X}$-ray beams with high brilliance, wide energy range, and coherence. The high-flux synchrotron source and the implementation of the micro/nano-sized beam make it possible to study energy materials from macroscopic device structures to microscopic single particle evolutions. When combined with X-ray spectroscopies, $\mathrm{X}$-ray can also probe chemical properties such as oxidation state and local atomic environment, as well as the structural and morphological information. A powerful, emerging technique, coherent diffraction imaging (CDI), which is made possible by the coherent feature of synchrotron $\mathrm{X}$-ray sources, can give spatially resovled information of the crystal structure such as strain and lattice distortion of samples.

In this review, we give a comprehensive overview of major $X$-ray imaging techniques and recent examples of their application on energy materials. We will focus on X-ray projection imaging, transmission $X$-ray microscopy (TXM), scanning transmission X-ray microscopy (STXM), tender and soft X-ray imaging, and coherent diffraction imaging (CDI), which have been developed to provide insights on the morphological, chemical, and crystalline properties of materials. Among these methods, STXM uses soft X-rays $(<2 \mathrm{keV})$, tender and soft $\mathrm{X}$-ray imaging use both tender ( $2-5 \mathrm{keV}$ ) and soft $\mathrm{X}$-rays, and TXM, X-ray projection imaging, and $\mathrm{CDI}$ use hard $\mathrm{X}$-rays (> $5 \mathrm{keV})$. The $X$-ray imaging techniques are based on different imaging principles including transmission (X-ray projection and TXM), fluorescence (tender and soft $X$-ray imaging), and scattering (CDI); STXM can be conducted in both transmission and fluorescence modes. X-ray projection imaging and tender and soft $X$-ray imaging have resolutions from several $\mu \mathrm{m}$ to tens of $\mu \mathrm{m}$, while the resolutions of TXM, STXM, and CDI are about tens of $\mathrm{nm}$. In the following sections, we will have a more detailed discussion about the working principles, recent progress, and scientific cases of each technique. 
We hope this review will help inspire the interest and broaden the view of readers and encourage more applications of synchrotron imaging techniques.

\section{X-ray projection imaging}

$X$-ray projection imaging is defined as the imaging form in which a sample is placed in between an $\mathrm{x}$-ray source and an $\mathrm{x}$-ray intensity detector. There is no other $\mathrm{x}$-ray optics after the sample. X-rays transmit through the sample then directly project onto the detector. Such imaging mode is different from other modes that will be discussed in the following sections either on the detection signal ( $x$-ray absorption) or $\mathrm{x}$-ray optics (no x-ray optics), as shown in Figure 1 (a). Imaging relies on the contrast between different parts of a sample to resolve its structures. Absorption $X$-ray imaging relies on the attenuation of an incident $X$-ray beam to form a projection image on a detector. The attenuation of $\mathrm{X}$-ray beam by matter follows Beer-Lambert law, which depends on the attenuation coefficients of the matter. With a partially coherent $\mathrm{X}$-ray source, which is the case with synchrotron sources and some modern X-ray lab sources, "phase contrast" imaging can also be applied to materials that are weakly absorbing. This takes advantage of the phase shift in a propagated X-ray beam as it passes through a sample, which is more sensitive than absorption contrast but requires extra measurements or data processing or both. It has been proved particularly useful for imaging low atomic number materials for batteries, with Ref [9-11] being notable examples.

Over the past ten years, the increasing sophistication of X-ray imaging tools has delivered increasing spatial and temporal resolutions, using both synchrotron and laboratory sources, along with $\mathrm{X}$-ray source and detector technology developments; consequently X-ray imaging has found applications in range of energy conversion and storage materials. This section explores the development and applications of projection $\mathrm{X}$ ray imaging to applications in energy materials.

Haibel et al. [12] were the amongst the first to openly publish X-ray tomography of battery architecture in their 2010 study of alkaline batteries. The first images with sufficient resolution to resolve the electrode architecture for a Li-ion battery were presented by Shearing et al [13], which provided the first insight into the heterogeneous microstructure of conventional graphite battery electrodes by quantifying a range of parameters including porosity, tortuosity and surface area. Subsequent studies across multiple length scales $[10,14,15]$ have further highlighted the hierarchical nature of these complex electrodes, observing heterogeneities across the nano-meter to micron scale. Extensive work has also been carried out to quantify battery architectures, particularly in response to battery failure, indeed the use of tomography as a post-mortem diagnostic is now considered routine [16], and has been widely applied in failure investigations (cf. Samsung Galaxy Note 7, Boeing Dreamliner etc). The combination of tomography data with various modeling platforms has quantified the relationship between performance and microstructure: for example the use of tomographic data as a framework for tortuosity calculation is now widespread [1720], indeed open-source software platforms are now freely available to facilitate this [21,22]. Other simulation approaches enable description of various phenomena, including chemo-mechano effects [23].

With the advent of ultra high-speed imaging at synchrotron sources, X-ray radiographic and tomographic imaging can be applied for in situ and operando battery characterizations. Operando studies using 
radiography and tomography have been conducted on commercial 18650 cells (Figure 1 (b)), yielding insights into the thermal runaway of lithium-ion batteries [24,25]. X-ray tomography has also been applied to explore the changes to electrode architecture as a function of state of charge [26] (Figure 1 (c)) and applied mechanical load. These 'four-dimensional' techniques (three spatial + time) have in particular yielded interesting results to guide the development of alloying type electrodes (Sn, $\mathrm{Si}$ ) and Li/S developments. [11,27-30] The application of digital volume correlation tools has been demonstrated as an effective means to quantify the local change in these 'time-lapse' images, which can identify strain within heterogeneous electrodes [28,31,32]. Besides Li-ion batteries, X-ray imaging has been widely applied to study other emerging battery chemistries including Na-ion, Li-sulfur, and Zinc [33-35].

(a)

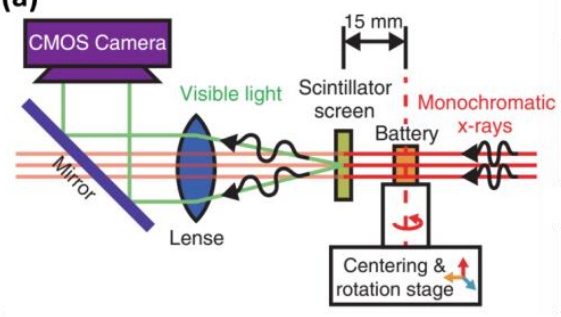

(b)

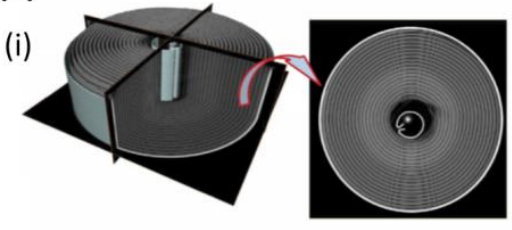

(ii)

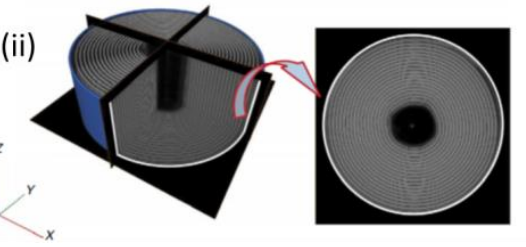

(c)

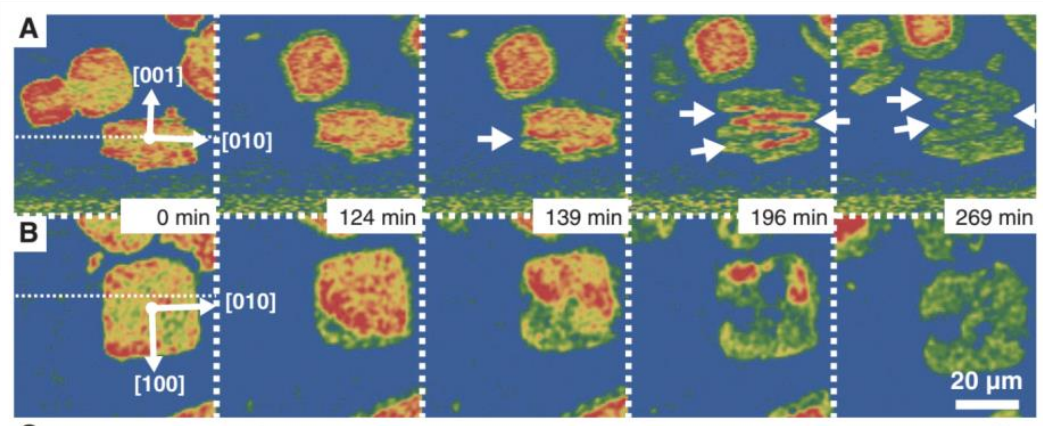

$$
\text { C }
$$
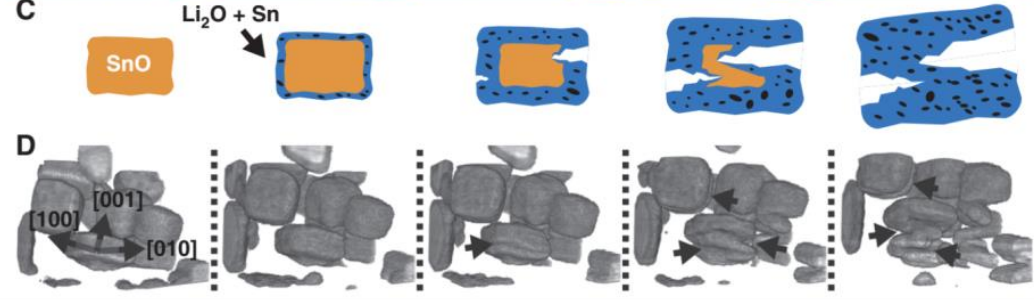

Figure 1. (a) Sketch of X-ray projection imaging setup. (b) 3D reconstructions of commercial LG 18650 NMC batteries: (i) 2.6 Ah battery containing internal cylindrical structural support with inherent safety advantages; (ii) 2.2 Ah battery with out the internal support. (c) Evolution of Sn electrode particle structure. Cross sections of coronal (A) and transverse (B) plane through a particle during electrochemical reduction. Horizontal dotted white lines in the cross sections at 0 min indicate cutting planes. (C) Schematic of particle phase evolution and crack growth leading to zig-zag morphology. (D) 3D rendering of subvolume visualizing zig-zag morphology in multiple particles. (a) and (c) are reproduced with permission from Ref. [26]. Copyright 2013, American Association for the Advancement of Science. (b) is reproduced with permission from Ref. [25].

Compared with heavy elements, element with low atomic number such as carbon $(Z=6)$ and lithium $(Z=3)$ are weakly attenuating for hard X-rays, resulting in low contrast between these and the electrolyte-filled pore space. With emerging phase contrast technique, $\mathrm{X}$-ray microtomography can enhance the contrast between different weakly attenuating materials such as carbon and lithium, though it usually needs more complicated setups and is typically more prone to different types of artefacts. Paul et al. successfully enable in-line phase contrast imaging lithium dendrite with a monochromated beam at $19 \mathrm{keV}$ with a bandwidth 
of $0.1 \mathrm{eV}$, where attenuation and refractive effects are decoupled to image interfaces between materials of similar attenuation [9] (Figure 2 (a)). Similarly, with a higher-flux synchrotron X-ray source at 2-BM beamline at Argonne Photon Source, lithium dendrite growth was directly imaged at 3D with a spatial and temporal resolution of $0.65 \mu \mathrm{m}$ voxels (edge length) and 1.8 seconds between each 3D reconstruction [36]. A similar work was also done at Advanced Light Source to successfully detect lithium dendrite growth underneath the polymer/electrode interface [37] (Figure 2 (b)). Similar to lithium, graphite has a weak X-ray attenuation and renders the poor contrast between graphite and other electrode components such as pores, binder and electrolytes in battery electrodes. With propagation-based phase contrast technique, an operando microtomography of weakly attenuating matters in electrochemical cycling has been demonstrated, and quantify microstructural dynamics of graphite in battery anodes [32].

(a)

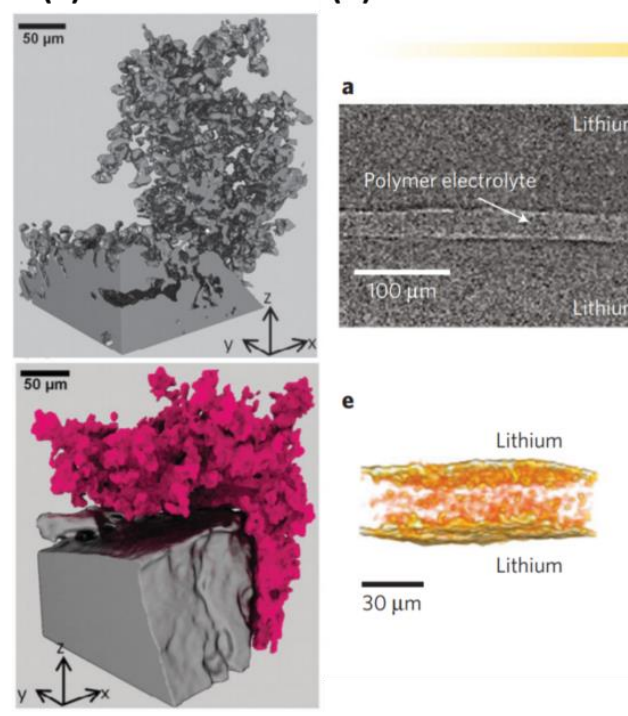

(b)

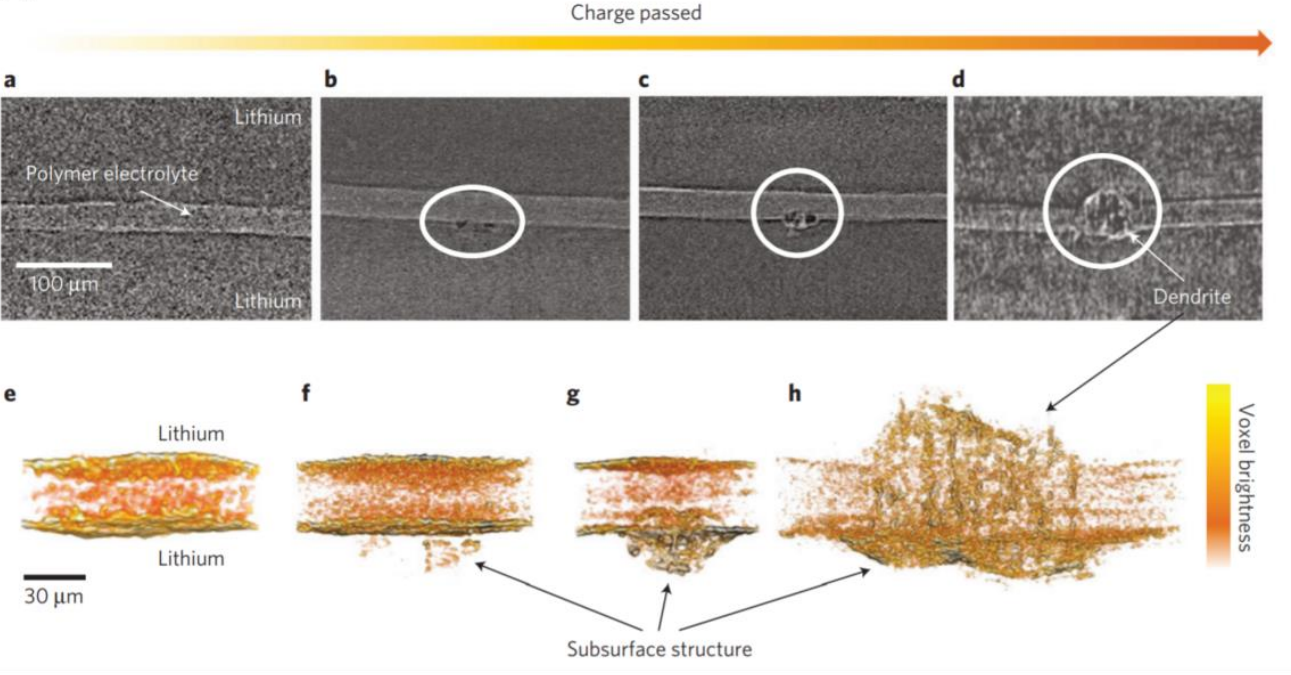

Figure 2. (a) 3D rendering of Li microstructures under a constant current (top), and higher density microstructures following plating and stripping (bottom). Reproduced from Ref. [9] with permission from The Royal Society of Chemistry. (b) Li dendrite growth with charge passed. a-d, X-ray tomography slices showing the cross-sections of symmetric lithium cells cycled to various stages. The thin, bright horizontal strip through the center of the images is the polystyrene-block-poly(ethylene oxide) copolymer electrolyte sandwiched between two lithium metal electrodes. $\mathrm{e}-\mathrm{h}$, Magnified, 3D reconstructed volumes of cells shown in the top panel. Reproduced from Ref. [37]. Copyright 2013, Springer Nature.

In addition to active materials, the capability of imaging ion transport is also crucial for battery electrodes, which is particular true for solid state batteries. It is well known that a number of interfaces occurs in a solid-state battery, including grain-grain, grain-void, grain junctions and electrode-electrolyte, etc. The physical characteristics and nature of these interfaces significantly affect lithium ions, electron and mass transport within a solid-state battery. With the synchrotron X-ray microtomography, Shen et al. imaged the structural transformation in Granet-type $\mathrm{Li}_{7} \mathrm{La}_{3} \mathrm{Zr}_{2} \mathrm{O}_{12}$ (LLZO) solid state electrolyte and investigated the effect of pore connectivity on lithium dendrite propagation [38] (Figure 3 (a)). Due to the presence of heavy Z-elements in LLZO, the attenuation length for the monochromatic beams is limited. Instead, the polychromatic white beam at high energy ensure good contrast between the ceramic and void region 
(pores and Li metal). The microstructural and tortuosity effects in LLZO solid electrolytes can be evaluated (Figure 3 (b)), which provides new insights into optimizing porosities and tortuosity for high-performance solid state batteries [39].

(a)

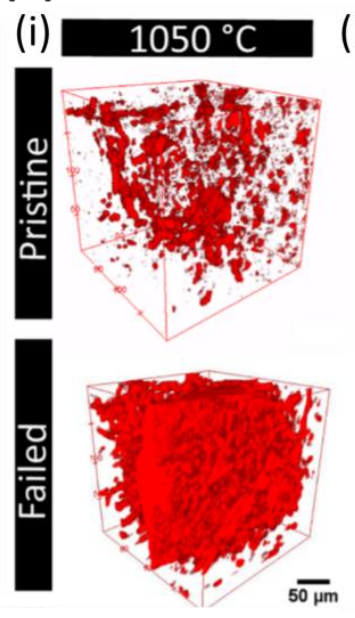

(ii)

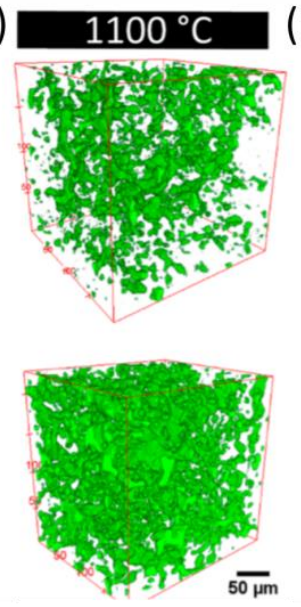

(b)

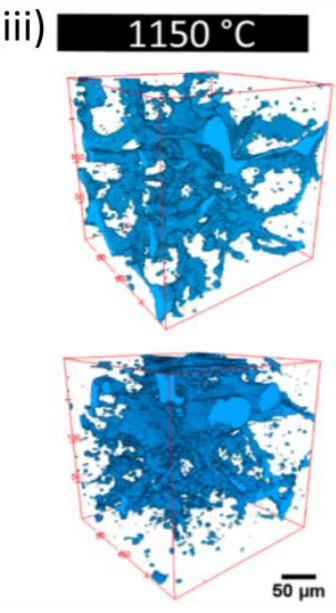

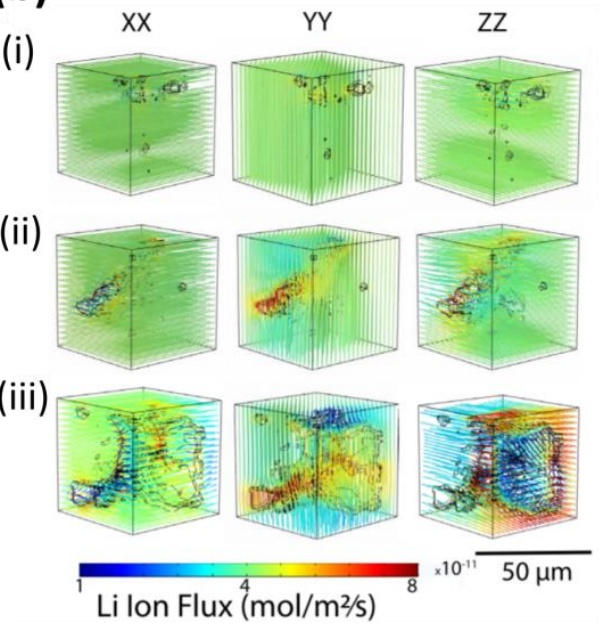

Figure 3. (a) Morphological changes of LLZO solid electrolytes sintered at (i) 1050 , (ii) 1100 , and (iii) $1150{ }^{\circ} \mathrm{C}$, obtained by X-ray tomographitc reconstructions. Reprinted with permission from Ref. [38]. Copyright 2018 American Chemical Society. (b) Li-ion flux flux streamlines along three principal axes for microstructural domains of real LLZO electrolytes sintered at (i) 1050 , (ii) 1100 , and (iii) $1150{ }^{\circ} \mathrm{C}$. Reprinted with permission from Ref. [39]. Copyright 2019 American Chemical Society.

$X$-ray projection imaging has also been used in fuel cell research works, most notably to polymer electrolyte membrane fuel cell (PEMFC). The first tomographic studies of gas diffusion layers emerged around 2006 [40], providing much needed microstructural information to inform numerical simulation of transport properties [41,42]. Much of works along this line have been summarized in an early review article by Bazylak [43] and a more recent review article by Zenyuk [44]. In complement to neutron radiography, the PEMFC community quickly realized the benefits of operando studies to explore water formation, which has now become a staple technique for fuel cell characterization [45-49], and has been used to evaluate processing parameters [50]. More recently, this has also been extended to evaluate the performance of polymer electrolyte based electrolysers for hydrogen production [51-53], providing insight into mutli-phase flow.

The stable of X-ray tomography techniques now available to the energy materials community enables routine multi-scale characterization of a range of devices for energy conversion and storage, which can be successfully correlated with other microscopy modalities to provide a comprehensive understanding of the hierarchical materials microstructure characteristic of many energy materials. Whilst X-ray projection imaging has merit of nondestructive detection of dynamic morphological structure evolution in large volume with high temperoal resolution, it has certain limitations. The typical spatial resolution of X-ray projection imaging is about $1 \mu \mathrm{m}$. This is not high enough to detect the structural changes, e.g., at single electrode particle level in lithium-ion batteries. It is also not able to detect crystallographic structure and 
chemical state distributions in a sample, which are both important in energy storage material studies. Therefore, other X-ray imaging modalities are needed. These techniques and applications will be discussed in details in the next sections.

\section{Transmission X-ray microscopy}

The operation principles of Transmission X-ray Microscopy (TXM) are similar to optical microscopy except that it uses hard $X$-rays instead of visible light to illuminate the sample. Figure 4 (a) shows the experimental setup of TXM. The X-ray beam is focused on the sample, and then the transmitted X-ray beam is magnified by a Fresnel zone plate working as the objective lens and projected onto the detector. TXM is a full-field imaging technique with the field of view set by the focused $X$-ray beam size and the spatial resolution determined by the width of the outmost zone of the zone plate. The typical resolution of TXM is about 20 - $30 \mathrm{~nm}$ [54-57]. The high-penetrative and non-destructive hard X-rays beam and the high-flux of synchrotron X-ray sources make TXM capable for in-situ/operando characterizations of energy materials. Furthermore, the TXM technique has well-developed hardware instruments [58,59] and software for data analysis $[60,61]$. We will discuss in the following sections how TXM has been applied to acquiring 2D and 3D morphological and chemical mappings in energy materials.

(a)

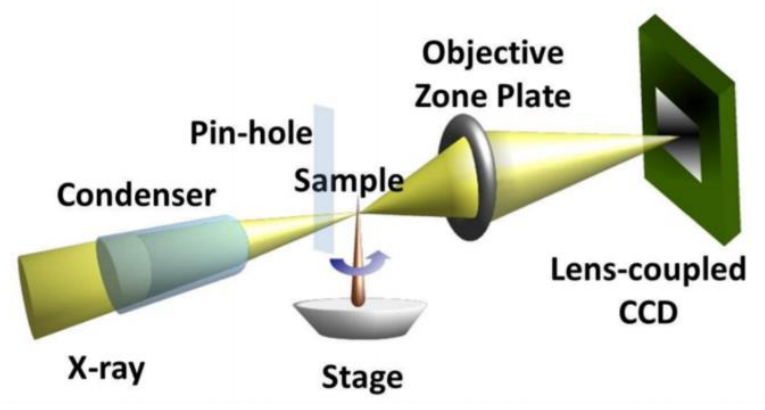

(b) Spectroscopic Image Series

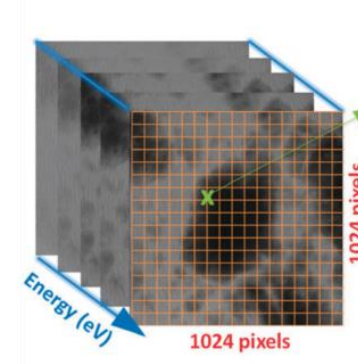

Figure 4. (a) Illustration of TXM experimental setup. (b) Working principles and data of TXM-XANES experiment on $\mathrm{CuO}$ anode. Reproduced from Ref. [54] with permission from The Royal Society of Chemistry.

\section{D Morphological and Chemical Mapping by TXM}

TXM can be conducted at single X-ray energy to provide morphological information from absorption contrast, or at multiple photon energies coupled with spectroscopy to show elemental and oxidation state distributions. Using single X-ray energy shortens data acquisition time, and increases the temporal resolution of operando experiments. Operando TXM using $6 \mathrm{keV}$ X-ray energy has been applied to measure the composition and morphology change of sulfur/Super P carbon composite particles during the cycling of Li-S battery [62]. The field of view (tens of micrometers, can be increased to millimeters by stitching images together) and the spatial resolution of TXM are suitable for the characterization of nano to microsized particles and agglomerates [54,57,62-64]. Nelson et al. were able to track individual particles and monitor the loss of polysulfides into the electrolyte by the contrast change between composite particle and electrolyte background during the operation of the battery, and have revealed that the bulk of polysulfides 
remain trapped within the sulfur/Super $\mathrm{P}$ composite particle matrix during the first electrochemical cycle. The operando results eliminate any artifacts that otherwise would be introduced in the system during posttreatment.

As aforementioned, TXM can provide 2D chemical mapping in addition to the structure and morphology image when coupled with X-ray absorption spectroscopy (XAS). This is achieved by taking TXM images at multiple $X$-ray energies across the element absorption edge. It is more time-consuming and has higher technical requirements than single energy TXM since many motors need to move to refocus the sample when the energy varies. A simplified method is the dual-energy contrast imaging technique [61], where TXM images are recorded at two energies below and above the absorption edge of the element of interest. An elemental map without oxidation state information can be obtained by quantifying the amount of element at each pixel from the edge jump [63]. A more powerful technique is the TXM-XANES imaging (XANES is short for X-ray absorption near edge structure). TXM images are collected at a series of X-ray energies along the XANES region, so a XANES spectrum can be generated at each pixel. Fitting the XANES spectra gives information on the quantity and oxidation states of the element that we want to probe at each pixel, thus a 2D chemical phase map can be obtained. Figure 4 (b) shows an example of TXM-XANES data collection on $\mathrm{CuO}$, an anode material for Li-ion battery. A series of TXM images are taken along the $\mathrm{Cu}$ K-edge, and a $1024 \times 1024$ array of spectra are generated, giving a spectroscopic map. Then the chemical phase information at each pixel is obtained through linear combination fitting of the spectrum from standard $\mathrm{Cu}, \mathrm{Cu}_{2} \mathrm{O}$, and $\mathrm{CuO}$ spectra, creating a 2D phase distribution map. By tracking the chemical phase transformation and composition of $\mathrm{CuO}$ particles during (de)lithiation, the authors have revealed that a size-dependent core (CuO)-shell ( $\mathrm{Cu}$ ) lithiation-delithiation mechanism of $\mathrm{CuO}$ anodes during the conversion reaction.

TXM-XANES is crucial in characterizing samples with no significant change in X-ray absorbance during the reaction. For instance, the high-density battery cathode materials such as layered metal oxides $\mathrm{LiMO}_{2}$ ( $M=\mathrm{Ni}, \mathrm{Co}, \mathrm{Mn}$, etc.) and olivine-phase $\mathrm{LiFePO}_{4}$, show little contrast change before and after Li intercalation, but the lithiation state can be obtained by the oxidation state of transition metals. Additionally, the K-edge of these transition metals used in battery cathodes almost all fall into the hard X-ray region (> 5keV), making in-situ/operando study easier with the high-penetration hard X-rays. Figure 5 (a) shows a recent study on $\mathrm{Li}_{x} \mathrm{Ni}_{0.6} \mathrm{Mnn}_{0.2} \mathrm{CO}_{0.2} \mathrm{O}_{2}$ (NMC-622) cathode particles, where the lithiation content in the cathode is indicated by the Ni K-edge position. NMC-622 particles are chemically and electrochemically delithiated to different SOCs with a similar degree of heterogeneity within the particles, as demonstrated by the Ni K-edge map (Figure 5 (a)). In addition, despite the relatively slow rate $(0.1 \mathrm{C})$ and long relaxation time in the electrochemical process, the heterogeneity of SOC still exists in almost every secondary particle. The authors proposed several causes of this inhomogeneity, including the liquid penetration limits and the anisotropic changes in lattice parameters. Another example is the operando TXM-XANES study on chemical phase evolution of LFP nano- and micro-sized particles during electrochemical cycling, as shown in Figure 5 (b). Significant inhomogeneity across the nano-sized LFP electrode at fast charging rate (5C) has been demonstrated, showing a non-uniform phase transformation. In addition, experiments on micro-sized LFP particles have shown intra-particle heterogeneity which supports the two-phase reaction model in LFP particles. This study highlights the importance of uniform electrode on battery rate performance. 
(a)

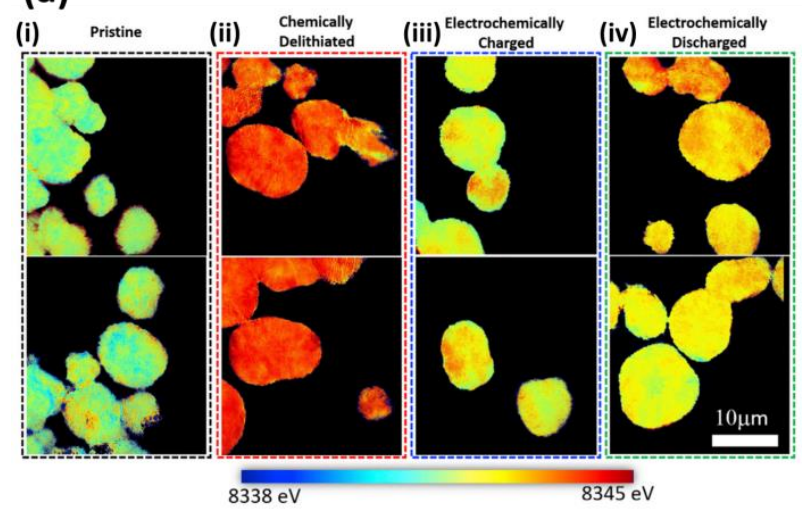

(b)

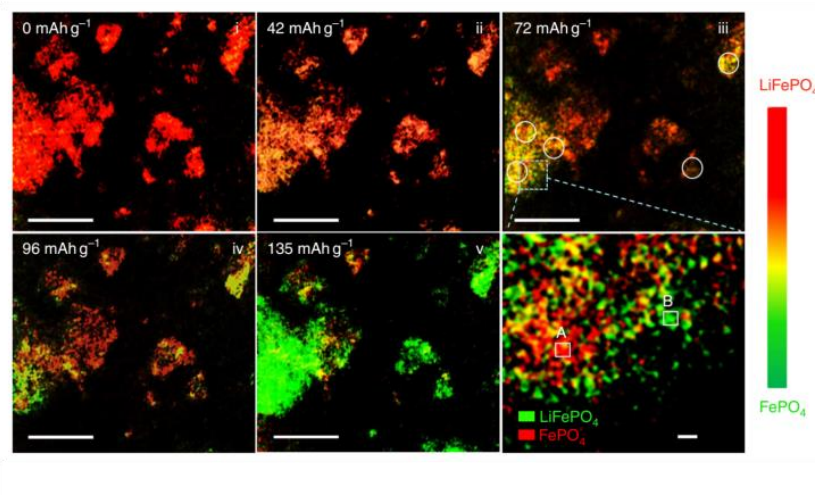

Figure 5 (a) 2D mapping of Ni k-edge of different samples: (i) pristine NMC-622; (ii) chemically delithiated NMC-622; (iii) electrochemically charged and (iv) discharged NMC-622. Reproduced with permission form Ref. [65]. Copyright 2019 Elsevier. (b) Chemical mapping of LFP electrode comprised of nano-sized (200-300 $\mathrm{nm})$ LFP particles, showing the inhomogeneous delithiation of LFP electrode at $5 \mathrm{C}$ fast charging rate. Reproduced with permission from Ref. [57]. Copyright 2014, Springer Nature.

\section{D Tomography imaging based on TXM}

X-ray tomography measurement is achieved by taking TXM projections of a rotating sample at each fraction of a degree. The 2D images collected at all angles are then reconstructed into a 3D image, providing the 3D structure and morphology information of the sample. The hard X-rays can penetrate through most of the outside cases of energy devices, enabling non-destructive 3D imaging of the inside components. With the high flux of synchrotron $X$-rays and the development of detectors for fast data acquisition, one tomography measurement could be carried out in several minutes, which makes in-situ/operando experiments possible $[66,67]$.

Compared with 2D TXM image which is a projection through the entire sample, the 3D structure analysis of X-ray tomography with tens of nanometer resolution has the ability to detect the cracks, porosity, and tortuosity within the sample. Figure 6 shows the X-ray tomography study on the 3D morphology evolution of Sn anode during (de)lithiation and (de)sodiation, demonstrating the different failure mechanisms between Li-ion and Na-ion batteries. [66,67] Quantitative comparison of the pores, cracks, fracture, and pulverization show that large $\mathrm{Sn}$ particles experience volume expansion and cracking during $\mathrm{Na}$ ion insertion, but there is only negligible pulverization during $\mathrm{Na}$ ion extraction. In contrast, the structural failure in Li-ion battery mainly occurs during the Li-ion extraction process when an enormous particle pulverization takes place, as shown in Figure 6 (a). 

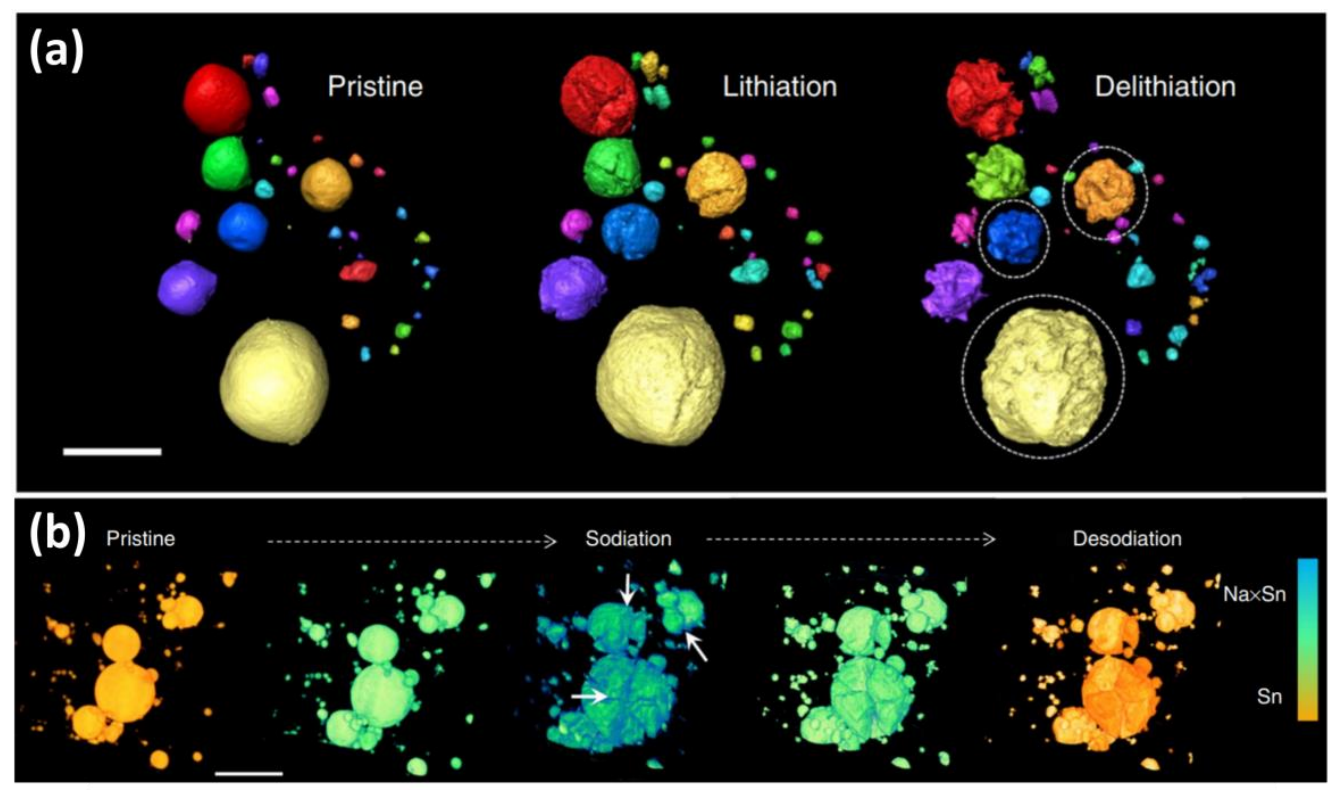

(c)

Li ion batteries

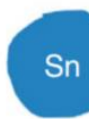

Lithiation
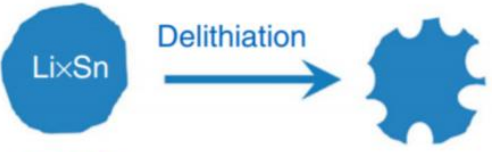

$\mathrm{Na}$ ion batteries
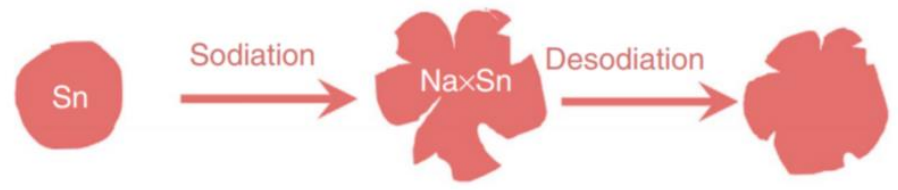

Figure 6. (a) 3D morphology evolution of Sn particles during the first lithiation-delithiation process. The colors on particles is only to assist in view. (b) 3D morphology change of Sn particles during the first sodiationdesodiation process. The colors represent the attenuation coefficient variation within the images, which indicates the phase change between $\mathrm{Sn}$ and $\mathrm{Na}_{x} \mathrm{Sn}$. (c) Schematic illustration showing the difference of $\mathrm{Sn}$ microstructural changes between (de)lithiation and (de)sodiation. Reproduced with permission from Ref. [66].

Similar to 2D TXM, tomography can be combined with XAS technique in order to obtain a 3D chemical mapping. In a recent 3D XANES study, tomography measurements with $30 \mathrm{~nm}$ resolution were conducted to study the delithiation of micro-sized $\mathrm{Li}_{1.3} \mathrm{Ta}_{0.3} \mathrm{Mnn}_{0.4} \mathrm{O}_{2}$ (LTMO) cathode particles [68]. The imaging data was collected at more than 100 energy points across the Mn K-edge to create a 3D chemical map, as shown in Figure 7 (a). The heterogeneity of the $\mathrm{Mn}$ valence status inside the particle is visualized, and a slice through the center of the particle shows that $\mathrm{Mn}$ is more oxidized on the particle surface than in the bulk. In addition, a large number of cracks are found in more delithiated LTMO particles with more oxidized Mn near the cracks, as shown in Figure 6 (b). This correlation suggests that the particle cracking mostly takes place on positions where the Mn oxidation state is saturated and oxygen is redox active. Through 3D tomography with depth resolution inside the particle, the reaction mechanism of LTMO cathode lithiation has been resolved and observed the chemomechanical interplay has been observed which plays an important role in battery failure mechanisms. 
(a)

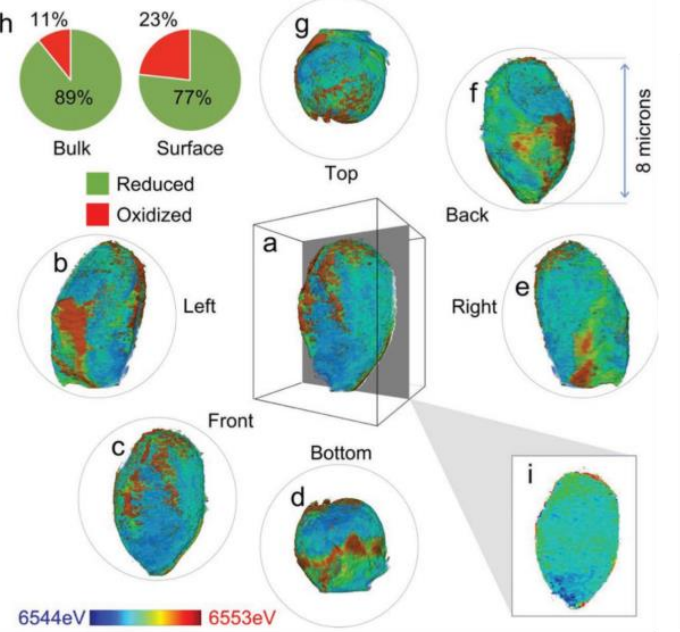

(b)

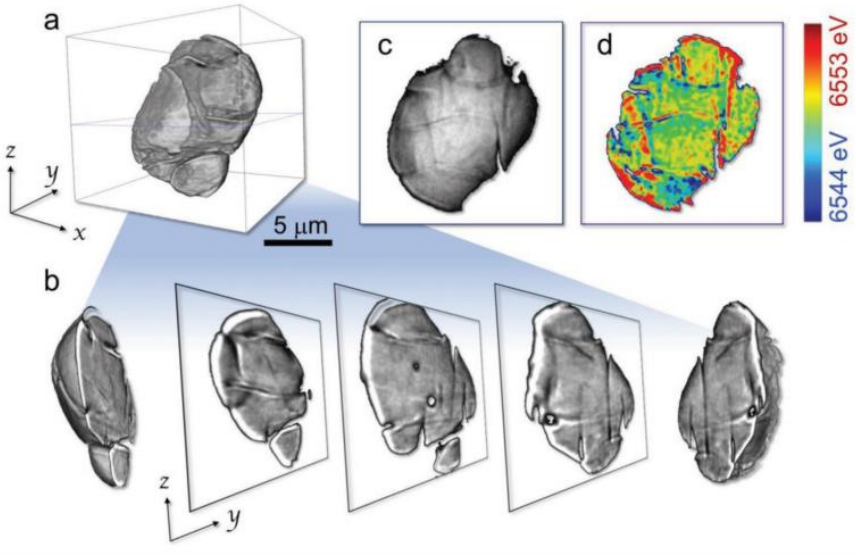

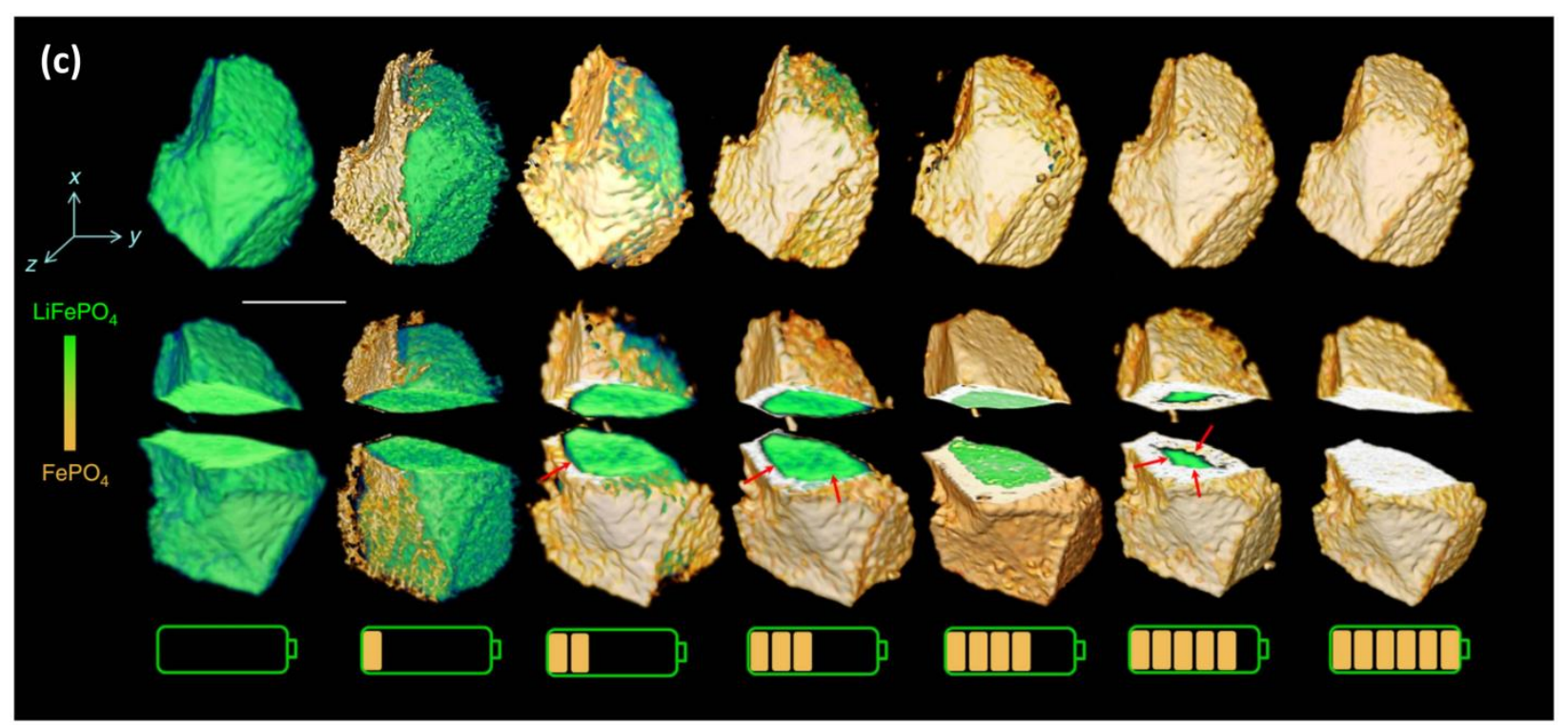

Figure 7. (a) a: 3D mapping of the Mn K-edge value of a $\mathrm{Lix}_{x} \mathrm{Ta}_{0.3} \mathrm{Mn}_{0.4} \mathrm{O}_{2}$ (LTMO) particle, showing the inhomogeneous delithiation state. b-g: The projection views in different orientations are also shown. h: the quantification of the phase segregation of the particle bulk and surface. i: a virtual slice through the center of the particle. (b) a: 3D morphology mapping of an LTMO particle at further delithiation showing the cracks. b: virtual slices through the particle at different depth. c: projective view of the particle. d: the Mn oxidation map of the projective view. (a) and (b) are reproduced with permission from Ref. [68]. Copyright 2019 Wiley$\mathrm{VCH}$. (c) 3D phase distribution mapping of a $\mathrm{LiFePO}_{4}$ particle as a function of charging time. The cut-away views reveal a change from anisotropic to isotropic phase boundary motion. (c) is reproduced with permission from [69].

Since one 3D chemical map includes the collection of TXM images at a $180^{\circ}$ rotation range and at various energies across element absorption edge, this massive dataset and long-time data collection make operando study challenging. With a self-developed operando X-ray tomography cell and carefully designed experiment, Wang et al. have successfully measured the operando XANES tomography during the lithiation of $\mathrm{LiFePO}_{4}$ (LFP) cathode [69]. The phase transformation is found to be anisotropic at the beginning of 
delithiation with a preferred phase boundary migration and becomes isotropic as the lithiation proceeds further (Figure $7(\mathrm{c})$ ). This so-called "5D" ( $x, y$, $z$, energy, and time) mapping experiment in model systems greatly helps to understand the reaction mechanisms of battery active materials, and the quantitative analysis methodology is broadly applicable for other energy materials.

\section{Scanning transmission X-ray microscopy}

Scanning transmission X-ray microscopy (STXM) uses a focused X-ray beam to scan across the sample and build up a microscopic image by recording the transmission intensity of X-rays at each position. STXM is mainly operated in the soft $\mathrm{X}$-ray range since it is easier to fabricate the high-resolution optics for soft $X$ rays. The spatial resolution is determined by the focused size of the $X$-ray beam and is typically $12-40 \mathrm{~nm}$ $[56,70]$. Soft $X$-ray experiments limit the sample thickness and are generally operated under vacuum, but also have advantages such as high resolution and being sensitive to light elements (carbon, oxygen, etc.). Similar to TXM, STXM allows for elemental and chemical mapping when coupled with spectroscopy technique [71-73]. Moreover, STXM has a flexible field of view and lower X-ray dose to the sample [56]. However, one drawback of STXM is the long acquisition time since the image is built up pixel-by-pixel by rasterizing the $\mathrm{X}$-ray beam across the sample, so the imaging time and the field of view are compromised.

One good example of operando STXM is the chemically mapping of $\mathrm{Li}_{x} \mathrm{FePO}_{4}$ single platelets by Lim et al. to track the spatial and temporal Li composition evolution during battery operation [72]. STXM images were taken across the Fe $L_{3}$ edge during $L i$ insertion and extraction with a liquid STXM imaging platform, so the Li composition $x$ at each pixel can be quantified from the Fe oxidation state. Chemical maps show fast and slow (de)lithiation domains within a single particle (Figure 8), indicating the non-uniform (de)lithiation pathway. Operando experiments show that there is little variation within the particle at high lithiation rates $(2 \mathrm{C})$, and domains of fast and slow lithiation are visible at intermediate rates $(0.2 \mathrm{C}$ to $0.6 \mathrm{C})$; while the exsitu mapping of half-lithiated LFP particles (Figure 8 (c)) show that the particles are phase separated into fully lithiated and fully delithiated domains with sharp phase boundaries. Moreover, the phase separation has been found to persist more during delithiation, which could be explained by the skewed current density vs. lithium content relationship.

In addition to the transmission signal, the chemical map can be obtained by the more elemental sensitive fluorescence yield (FY) [74,75], and the more surface-sensitive Auger electron yield (EY) [76]. Zhou et al. have measured the morphology and element distribution of Fe doped $L N M O\left(\mathrm{LiNi}_{1 / 3} \mathrm{Fe}_{1 / 3} \mathrm{Mn}_{4 / 3} \mathrm{O}_{4}\right)$ cathode using STXM in X-ray fluorescence (XRF) mode [75]. Unlike in transmission mode for soft X-rays where specimen with thickness less than hundreds of nanometers is required, XRF-STXM is less stringent on sample thickness and is more sensitive to low concentration elements. By mapping $\mathrm{Ni}, \mathrm{Mn}, \mathrm{Fe}$, and $\mathrm{O}$ distribution, $\mathrm{Ni}$ and Fe concentrations are found to vary more between facets and edges than the Mn and O concentrations. Additionally, the XANES mapping shows that (100) facet has more $\mathrm{Mn}^{3+}$ than the (111) facet, which is also confirmed by total electron yield (TEY)-STXM. This indicates a relative increase of disorder and more oxygen vacancies in the (100) facet. 


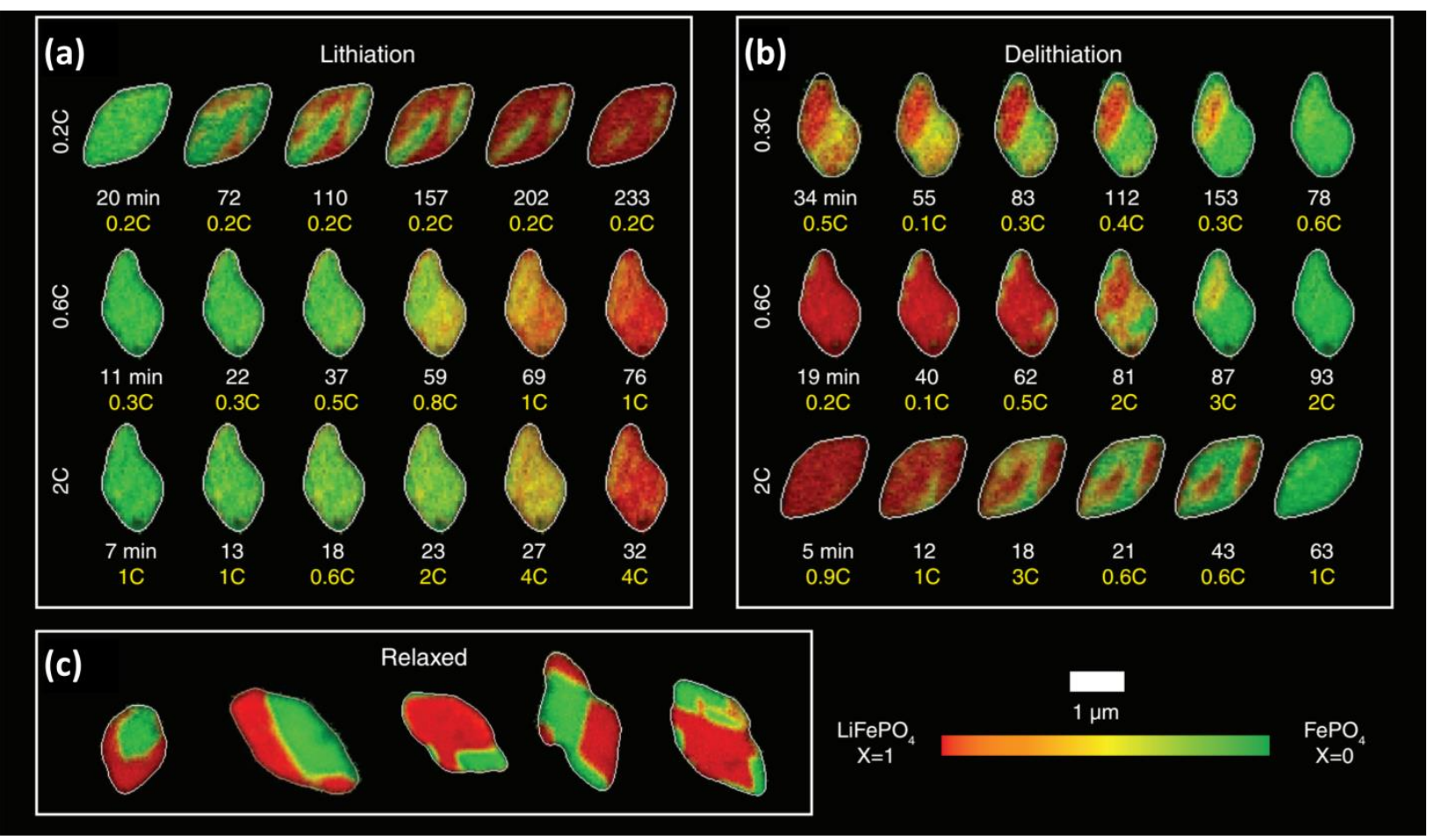

Figure 8. Representative frames of selected $\mathrm{LiFePO}_{4}$ (LFP) particles during Li insertion (a) and extraction (b) at different lithiation and delithiation rates from $0.2 \mathrm{C}$ to $2 \mathrm{C}$. (c) Ex-situ images of LFP particles showing the equilibrium distribution of $L i$ within particles. The hue represents the Li composition (green, $x=0 ;$ red, $x=1$ ). Reproduced with permission from Ref. [72]. Copyright 2016, American Association for the Advancement of Science.

\section{Tender and soft X-ray imaging}

While the capabilities of synchrotron-based microscopy already cover a vast photon energy range, spot size, and detection schemes with considerable sophistication as well as extensive applications, this section merely focuses on microscopy and relating techniques (e.g. microprobe) using tender and soft X-ray microbeams and X-ray fluorescence (XRF) detection with modest energy resolution.

Tender X-rays and soft X-rays are, in the context of this discussion, photons with energy in the range between $2 \mathrm{keV}$ to $\sim 5 \mathrm{keV}$, and $180 \mathrm{eV}$ (just below the B K-edge) to $2 \mathrm{keV}$, respectively. They fill the gap between vacuum ultraviolet, VUV (30 eV - $180 \mathrm{eV})$ and hard X-rays (> $5 \mathrm{keV})$. More recently, high spatial resolution (tens of nanometer) soft $X$-ray imaging has been realized using scanning transmission $X$-ray microscopy (STXM) [77], which typically covers the energy range $200 \mathrm{eV}-2 \mathrm{keV}$ with a submicron beam with a few exceptions in which energy extends to tender X-rays [78]. However, except for a few recent beamlines such as LUCIA at SOLEIL [79], PHOENIX beamline at SLS [80], and beam line 14-3 at SSRL, tender $\mathrm{X}$-rays $(1.7-5 \mathrm{keV})$ is not always covered by most light sources. Photons with these energy ranges were difficult to produce with enough intensity in the laboratory prior to the synchrotron era, and they are also challenging to the optics solution of the beamline since the $2 \mathrm{keV}$ range is at the high end of gratings and the low end of crystal monochromators. In addition, tender and soft X-ray beamlines, like their VUV 
counterparts, still require vacuum end station in most applications, although a He environment is possible for close distance operations.

In this review, emphasis is placed on the micro-imaging capabilities using KB mirror focusing on two unique beamlines at the Canadian Light Source (CLS) with imaging capabilities which one of the authors' team has developed - the Soft X-Ray Microanalysis Beamline (SXRMB) with an energy range of 1.7-10 keV [81-83] and the Spherical Grating Monochromator (SGM) beamline [84] with an energy range of $250 \mathrm{eV}$ ( just below the C K-edge) to $2 \mathrm{keV}$ (just above the Si K-edge).

These tender and soft X-ray microbeam capabilities are ideally suited for energy materials and environmental studies among many other applications. The SXRMB covers the Si, $\mathrm{P}, \mathrm{S}, \mathrm{Cl}, \mathrm{K}$ and $\mathrm{Ca}$ K-edge, and $4 \mathrm{~d}$ transition metals L-edges. It can be extended to the lower end of hard X-rays, covering first-row transition metals K-edges as an added advantage for imaging, for example, both Fe and $\mathrm{P}$ in $\mathrm{LiFePO}_{4}$, with K-edges at 7112 and $2146 \mathrm{eV}$, respectively, can be imaged at the same beamline, as illustrated below. Many energy materials are functionalized with these elements such as electrode materials in $\mathrm{Li}^{+}$(e.g. $\mathrm{LiFePO}_{4}$, Li$\mathrm{S}$, Li-P, etc.) and $\mathrm{Na}^{+}$(e.g. $\mathrm{Na}-\mathrm{O}_{2}$ ) batteries [1,85], electrocatalysts for fuel cell membranes [86], and solar cells (e.g. S in CZTS) [87] as well as light emitting flat panel display. Thus, imaging the a desired area of a device, an electrode, for example, and investigating the texture and the hot and cold spots using selected $\mathrm{X}$-ray excitation (e.g. at characteristic resonance above the edge) and X-ray fluorescence (XRF) detection will lead to in-depth understanding of the morphology-performance relationship and the role of the surface electrolyte interface (SEI), which in turn will help iterate the improvement of the device. In the following, the imaging capabilities of the SXRMB are discussed with examples to illustrate its performance followed by the SGM imaging capabilities, also with illustrations. A summary of the present status of these techniques will then be presented together with a note on the prospect of emerging capabilities and applications.

\section{Tender X-ray imaging and microprobe}

SXRMB is a bending magnet double crystal monochromator beamline equipped with two sets of interchanging crystals, InSb (111) and Si (111), for accessibility of Si K-edge and flux-resolution trade-off at higher energies, respectively [81-83]. Micro-focusing at the microprobe station is achieved using KB mirrors, taking in $0.15 \mathrm{mrad} \times 0.15 \mathrm{mrad}$ of the beam. The KB system and their control motors (bending and adjustments) are housed in a vacuum chamber. The sample stage is in a separate chamber downstream, equipped with a four-element Si (Li) drift detector (SDD), a CCD camera and a three-axis sample stage. For a typical scan, the spot size is $\sim 11.0 \mu \mathrm{m}(\mathrm{V}) \times 10.8 \mu \mathrm{m}(\mathrm{H})$ with a photon flux of $\sim 10^{9}$ photons $/ 100 \mathrm{~mA} / \mathrm{sec}$. With a pixel size of $15 \mu \mathrm{m} \times 15 \mu \mathrm{m}$ to $40 \mu \mathrm{m} \times 40 \mu \mathrm{m}$, and 1 second dwell time, it will take 2 to 3 hours to acquire an image of a $\sim 5 \mathrm{~mm} \times \sim 5 \mathrm{~mm}$ area.

This tender X-ray imaging performance is illustrated below in tracking the morphology and impurities of an ingot of $\mathrm{LiFePO}_{4}$ cathode synthesized from a large-scale process involving Si doping [88]. A primary issue with LFP is its intrinsic low electronic conductivity and lithium ion diffusion coefficient as well as long-term cyclability $[89,90]$. It is found that elemental doping, such as doping silicon into single crystal LFP leads to increased ionic conductivity and lower electronic conductivity [91]. 


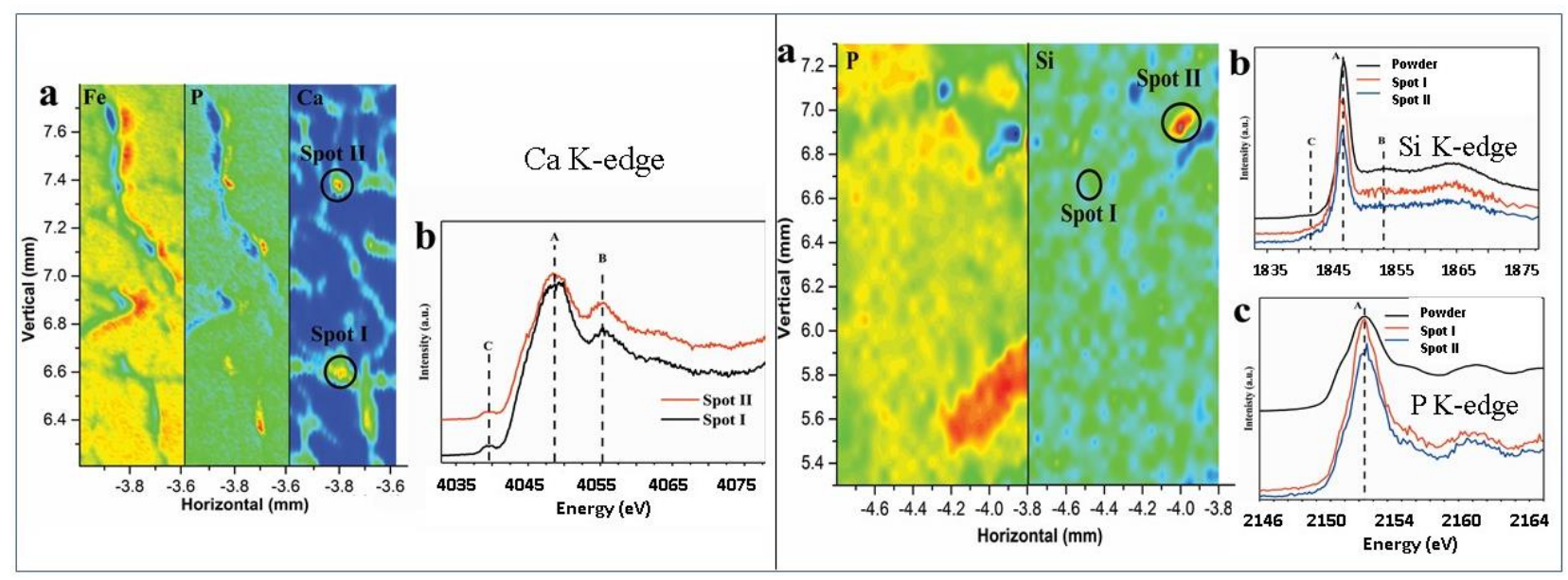

Figure 9. XRF map of Fe, $\mathrm{P}$, and $\mathrm{Ca}$ (left panel) and $\mathrm{P}$ and Si map (right panel) of a $\mathrm{LiFePO}_{4}$ ingot prepared with Si doping; corresponding micro-XANES and XANES of a powder sample are also shown. Requested with permission from Ref. [88]. Copyright 2019 Canadian Society for Chemical Engineering.

Figure 9 illustrates the Fe and P XRF maps along with the map of Ca as impurity from the precursor ( $a$, left panel, 7. 2 keV excitation, just above the Fe K-edge) and Si and P XRF maps (a, right panel, 2.2 keV excitation, just above the P K-edge) of an ingot of a given IOC (iron ore core) purity [88]. The left panel maps clearly demonstrate the non-uniformity of the LFP ingot. Based on the concentration of $\mathrm{Fe}, \mathrm{P}$, and $\mathrm{Ca}$, two unique regions are selected for micro-XANES analyses. The first region contains both Fe and $\mathrm{P}$, with a very low concentration of $\mathrm{Ca}$, which is the majority phase (blue region in Ca map). The second region has a high concentration of $\mathrm{P}$ and $\mathrm{Ca}$ with low Fe. Ca K-edge XANES in both regions closely resemble those of $\mathrm{Ca}$ containing glass such as $\mathrm{CaSiO}_{3}$ and Anorthite $\left(\mathrm{CaAl}_{2} \mathrm{Si}_{2} \mathrm{O}_{8}\right)$. A qualitative comparison between the data and literature indicates a relatively high degree of disorder in the impurity phases [92]. The Si and P XRF maps on the right panel were collected for the same specimen excited at a photon energy of $2.2 \mathrm{keV}$ tracking both $\mathrm{P}$ and Si distribution. The Si and $\mathrm{P}$ maps again show non-uniform distribution. Although Si doping in LFP lattice is expected to be substitutional at the P site and P and Si XRF maps are expected to show high correlation. This is not observed, most due to the presence of impurities in the form of $\mathrm{Li}_{3} \mathrm{PO}_{4}$ (confirmed by XRD) and silicon-containing glasses (formed during the melt-synthesis process). Si K-edge micro-XANES (b) at the region with low Si concentration (Spot I) and high concentration region (Spot II) have been compared to a powder sample. In both regions, the white line (peak A) has the same profile as the powdered sample. The Si K-edge micro-XANES at Spot II shows high Si concentration with cristobalite spectral character but no indication of cristobalite structure is seen in Spot I. The P K-edge XANES (c) in these two regions show similar features as Si-doped in LFP powdered samples, confirming the dominant presence of LFP as the main constituent.

\section{Soft $X$-ray imaging and microprobe}

Upon the recent installation of KB mirrors, the SGM at CLS has since provided soft X-ray microprobe capabilities not readily available in most synchrotrons. The SGM is an undulator-based, Dragon-type design and is equipped with 3 gratings covering the whole range of ( $250 \mathrm{eV}-2 \mathrm{k} \mathrm{eV}$ ) with ultrahigh energy resolution ( $>10,000$ at the N K-edge) [84]. The KB mirror provides a beam size of $\sim 10 \mu \mathrm{m} \times \sim 10 \mu \mathrm{m}$, with flux on the 
order of $10^{10}$ photon/sec during routine operation. XRF mapping is conducted with a fixed focused beam. The specimen, instead of stepping, is slewed continuously line by line. The elemental fluorescence signal is captured by an SSD detector (Amptek, fast SDD with CUBE preamp) which has a modest energy resolution of $\sim 80 \mathrm{eV}$ at the $\mathrm{O}$ K-edge $\left(\mathrm{E}_{\mathrm{o}} \sim 543 \mathrm{eV}, \mathrm{O} \mathrm{K}_{\alpha}\right.$ at $\left.\sim 525 \mathrm{eV}\right)$.

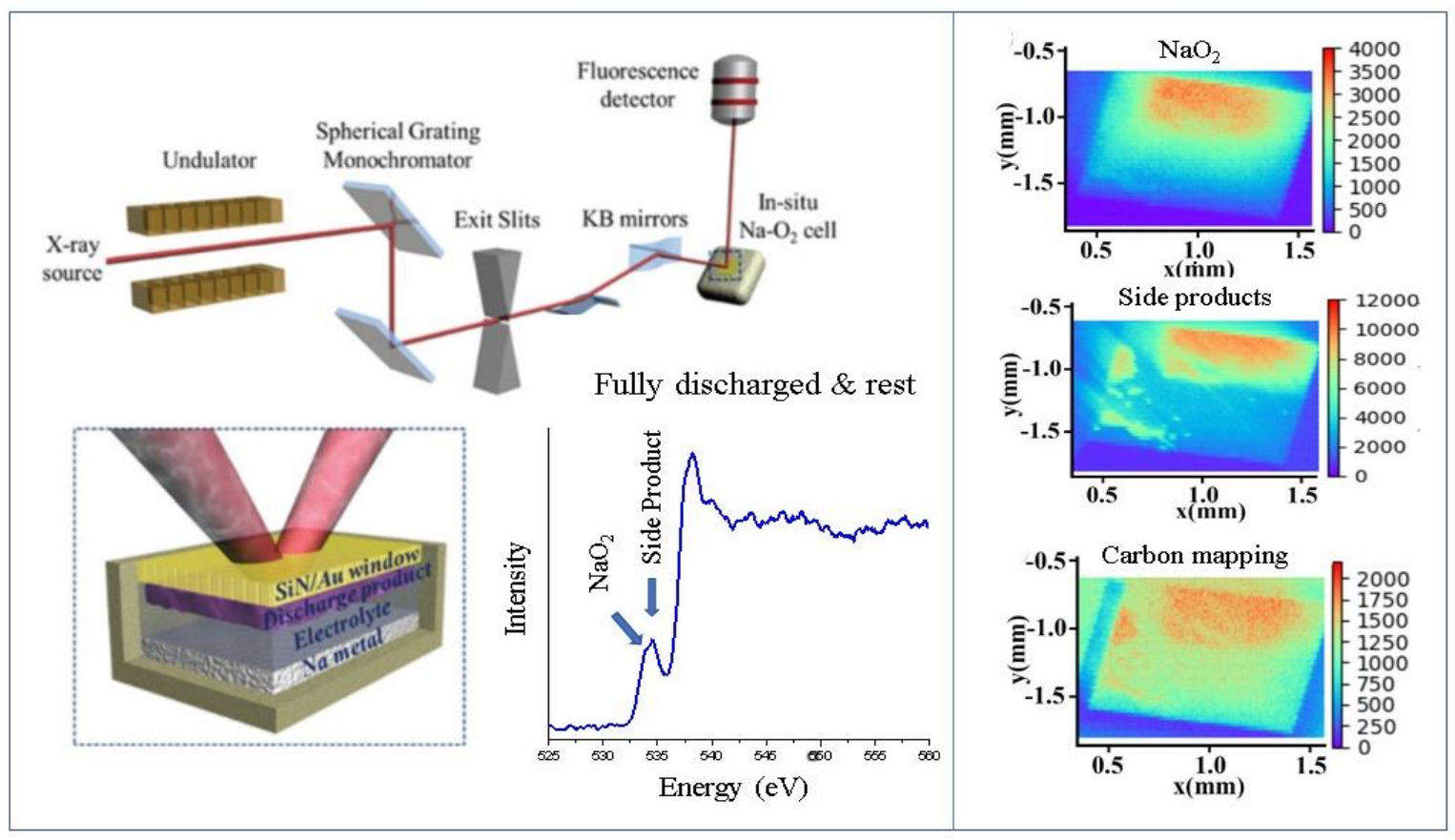

Figure 10. Top left panel: Schematic of SGM beamline at CLS and the soft X-ray XRF mapping and microprobe end station. Bottom left panel: $\mathrm{Na}-\mathrm{O}_{2}$ electrochemical cell for in operando measurements (left) and O K-edge XANES of Na- $\mathrm{O}_{2}$ battery at the full charge state and rest (right). Right panel: $\mathrm{O}$ and CXRF map of the electrode excited at the selected $\mathrm{O} \mathrm{K}$-edge resonance of $\mathrm{NaO}_{2}$ and side products as marked (see text). Reproduced from Ref. [93] with permission from The Royal Society of Chemistry.

Figure 10 shows the beamline layout and the $\mathrm{O}$ K-edge excited $\mathrm{O} \mathrm{K}_{\alpha}$ and $\mathrm{C} \mathrm{K}_{\alpha} \mathrm{XRF}$ mapping of the $\mathrm{Na}$ electrode in an in situ, in the operando measurement of a $\mathrm{Na}-\mathrm{O}_{2}$ electrochemical cell inside the vacuum chamber [92]. A $\mathrm{Si}_{3} \mathrm{~N}_{4}$ window separates the cell from vacuum yet allows the incident and fluorescence photon to come through (Figure 10, left panel, bottom left). One of the issues with $\mathrm{Na}-\mathrm{O}_{2}$ battery is the formation of side-products in $\mathrm{Na}-\mathrm{O}_{2}$ cells, resulting in increased charging over-potential as well as decreased Coulombic efficiency. Figure 10 (right panel) shows maps which track the chemical changes occurring in the discharge products formed in $\mathrm{Na}-\mathrm{O}_{2}$ cell in contact with the cell electrolyte. A fully discharged $\mathrm{Na}-\mathrm{O}_{2}$ cell was set to rest at an open circuit voltage. The $\mathrm{O}\left(\mathrm{K}_{\alpha}\right)$ XRF maps were obtained with excitation energy tuned to the $\mathrm{O}$ K-edge resonance characteristic of sodium superoxide, $\mathrm{NaO}_{2}(532 \mathrm{eV})$ and the side product ( $533.5 \mathrm{eV}$ ) as marked (Figure 10 left panel, right bottom). The details of the origin of the resonances have been discussed elsewhere [93], and in several relating works including a TXM study [94]. This SGM mapping not only confirms that $\mathrm{NaO}_{2}$ is a product in the operation of the $\mathrm{Na}-\mathrm{O}_{2}$ battery but also reveals the morphology of the electrode surface. It can be seen from the top right panel of Figure 10 that at the $\mathrm{NaO}_{2}$ resonance $\left(532 \mathrm{eV}\right.$ ), there is a gradient in the concentration of O XRF intensity across the $\mathrm{Si}_{3} \mathrm{~N}_{4}$ 
window, confirming the formation a thin film of discharge products of $\mathrm{NaO}_{2}$ over the gold-coated electrodes. The gradient is mostly due to specimen-detector geometry. However, at the side-product resonance $(533.5 \mathrm{eV})$, the XRF intensity shows several scattered islands of high concentrations, representing the more non-uniform formation of side products. $C K_{\alpha}$ map at $533.5 \mathrm{eV}$ excitation (c) shows similar distribution as the side-products, as tracked by O XRF.

The tender and soft X-ray capabilities at the $\mu \mathrm{m}$ and tens of $\mu \mathrm{m}$ spatial resolution described above are just the beginning as they started to provide services to the research community. It should be noted that these techniques can also work with micro-spectroscopy under in situ, in operando conditions, rendering both spatial and chemical information. They are filling the gap in the traditionally more challenging energy region. We are presently implementing KB capabilities at the variable line space plane grating monochromator (VLS_PGM) beamline at CLS to provide a $\sim 20 \mu \mathrm{m} \times 20 \mu \mathrm{m}$ microbeam for mapping in the VUV region (30 $\mathrm{eV}-250 \mathrm{eV}$ ) [95]. This energy is of particularly interest to the surface and near-surface region as VUV has very short penetration depth, providing the much- needed information which is often smeared out by bulk contributions in hard X-ray microscopy.

Returning to tender and soft X-ray microscopy and micro-spectroscopy. More can in fact be done with the present instrumentation, such as taking advantage of polarization of the incident photon delivered by elliptically polarized undulators, selecting a characteristic resonance for chemical speciation, and implementing X-ray emission spectrometers to improve the chemical sensitivity of XRF from modest tens, to hundred $\mathrm{eV}$ to $<1 \mathrm{eV}$ in the tender $\mathrm{X}$-ray, and $<100 \mathrm{meV}$ in the soft $\mathrm{X}$-ray region. Under favorable conditions, inversed partial fluorescence yield can also be used for imaging [96]. Finally, it should be noted that X-ray excited optical luminescence (XEOL) is another tool to image light emitting materials. Many materials, especially nanostructured semiconductors, inorganic and organic light emitting materials, can convert the X-ray they absorb into optical photons; the conversion process is directly connected to the morphology and crystallinity of the materials such as crystallite size, defects and impurities [96]. Since the $\mathrm{X}$-ray to optical photon conversion dynamics depends on the energy transfer process, of which, the time scale can span many orders of magnitude, from ps to $\mu$ s and beyond [97]. As the synchrotron pulse is typical $\sim 30 \mathrm{ps}$ and X-ray Free Electron Laser (XFEL) is 100 fs with tunable repetition rates, time-resolved imaging via different energy transfer channel is a possibility. Figure 11 below shows a couple of examples of XEOL imaging of rare-earth phosphors excited in the tender ( $\mathrm{Ce}$ and $\mathrm{Tb}_{\mathrm{L}}$-edge) and soft X-ray (O K-edge) region, respectively $[98,99]$. It can be seen from Figure 11 that XEOL with a given emission channel, has different depth and site selectivity than XRF hence it produces slightly different maps, left panel) and that an individual nanostructure can be mapped with optical yield (right panel). 


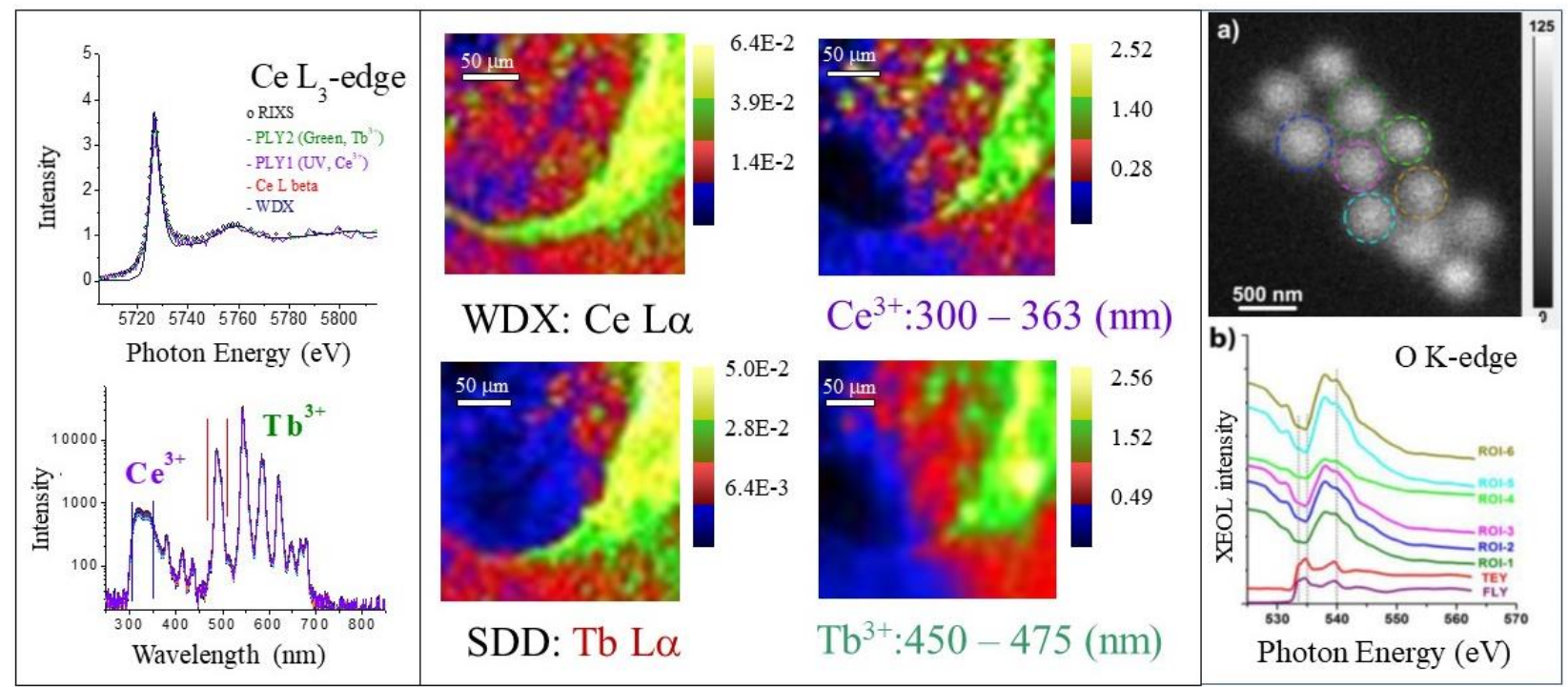

Figure 11. Imaging with $X$-ray excited optical luminescence yield, left panel shows the $\mathrm{L}_{3}$-edge absorption of a commercial rare-earth phosphor with X-ray and optical photon detections (top) as well as the XEOL spectrum (bottom); mid panel shows the 2D image with XRF (left) and optical photons (right) in the UV and blue originating from $\mathrm{Ce}^{3+}$ and $\mathrm{Tb}^{3+}$, respectively; right panel shows XEOL image from Eu doped $\mathrm{Y}_{2} \mathrm{O}_{3}$ nanoparticles (top) together with the nano-XANES at region of interest (ROI) color coded. The left and mi panels are reproduced from Ref. [98]. Copyright @ 2010, AIP Publishing. The right panel is reproduced from Ref. [99]. Copyright Microscopy Society of America 2018.

\section{Coherent Diffraction Imaging}

Coherent imaging methods are unique in that they dispense with the use of lenses to form images in exchange for exploiting coherence properties of the incident beam to form images from scattered light through computational algorithms [100]. In their purest form, coherent imaging methods directly measure the light scattered from an object. The assumption of coherence means that every scattered ray will have a fixed phase relationship to every other ray and thus form an interference pattern in the far field that is uniquely determined by the structure of the scattering object. Since only the intensities of the scattered waves can be measured, and not their relative phases, it is only possible to extract an image of the sample from the interference pattern if the lost phases can be computationally recovered and the waves back propagated to the sample plane to form the image of the sample.

Mathematically this coherent scattering process is described by the Fourier Transform, for which efficient computer algorithms are well established. It has been shown that the relative phases of the scattered waves can be retrieved directly from the intensities of the measured interference, or diffraction pattern if the pattern is sampled beyond the so-called spatial Nyquist frequency of the scattering object, which is given by its spatial extents. To achieve the inversion to an image there are iterative computational algorithms that are still a topic of development. However, in many applications, the algorithms and experimental methods are sufficiently developed to enable coherent $x$-ray diffraction imaging to impact scientific investigations [101-103]. 
There are two reasons why one turns to coherent $x$-ray diffraction imaging (CXDI) for materials characterization. One is the attempt to achieve very high spatial resolution imaging with highly penetrating $\mathrm{x}$-rays [104]. This is often challenging to achieve with lenses capable of focusing $\mathrm{x}$-rays and even more so with lenses adequate for image formation. Additionally, high resolution (tens of nanometers) $x$-ray optics typically have very short working distance making in-situ and operando imaging experiments challenging [105]. CXDI resolution is not determined by the optical system of the $x$-ray beam, but by the total angular extent to which $x$-rays are scattered, recorded, and subsequently phased and inverted to an image. Therefore, with modest $x$-ray optics producing spot sizes with hundreds of nanometer dimensions, one can image samples with a spatial resolution of tens of nanometers. Additionally, since coherent imaging is at its heart based on scattering, one can image a wide variety of contrast mechanisms, including amplitude and phase contrast due to the complex index of refraction of the material [106], elemental and chemical state contrast due to anomalous absorption of x-rays by core level photoelectron ejection [107] or in the case of Bragg diffraction, crystalline structure and lattice strain [108-110].

In recent years coherent imaging methods have divided along two paths. Those that require the object of interest fit entirely into the beam used to probe the sample, traditionally called Coherent Diffraction Imaging, and those that rely on scanning a sample of greater extent across a beam, with the requirement at the step sizes be less than the size of the beam used to probe the object $[100,111]$. The scanning version of coherent imaging is called Ptychography, from the Greek word meaning "to fold", implying the data from different regions of the sample are folded together in the computational algorithms used to produce an image. Ptychography studies in the small angle scattering regime have been conducted on ex-situ cathode materials. In Shapiro et al. a beautiful example of imaging a partially delithiated LiFePO4 particle is presented. This work had a contrast given by tuning the x-ray beam energy near an iron L-edge to gain sensitivity to chemical speciation. With this, they were able to image the contrast between LiFePO4 and FePO4 at $4 \mathrm{~nm}$ resolution also resolving cracks in the material and identifying enhanced pathways for lithium migration [107].

Bragg coherent $x$-ray diffraction imaging $(B C D I)$ is of the first kind of coherent imaging, requiring compact objects for the experiment. It is becoming a powerful technique for operando imaging of crystalline properties of materials, particularly for studies of functioning batteries [112-116]. An advantage of BCDI is that Bragg diffraction allows the technique to be specific to a single component of a heterogenous system. One can tune the instrumentation to detect diffracted signals from a single lattice spacing, characteristic of just the cathode material of an assembled battery for instance. So, while the $x$-ray beam will be passing through every layer of a fully functioning standard coin cell, one can focus on imaging the evolution of just a single crystalline component. In fact, using relatively high energy $\mathrm{x}$-rays, the Bragg peaks of individual randomly oriented crystals in the cathode will be sufficiently separated that a single crystal can be imaged, or several sequentially imaged during a charge/discharge cycle with a temporal resolution of tens of minutes. The only strict BCDI experimental requirement is that the objects of interest fit entirely within the $x$-ray beam used to probe the sample. The so-called compactness of the sample is a critical component of the iterative phase retrieval algorithms used to form images. Additionally, since the measurements are done in the vicinity of Bragg peaks, the method has inherent sensitivity to distortions of the crystalline lattice, which impart a minute phase onto the scattered beam and shows up in the retrieved images as a 
phase structure in a complex electron density distribution, where the amplitude is proportional to the magnitude of the density and the phase is directly proportional to the distortion of the lattice projected onto the Bragg peak of the measurement. The sensitivity to strain is on the order of $10^{\wedge}-4$ giving picometer sensitivity to distortions of the lattice on length scales determined by the accurate phasing of the coherent diffraction signal at high momentum transfer relative to the measured Bragg peak. The distortion field of the lattice is evident in the coherent diffraction pattern as a local asymmetry about the Bragg reflection, as is evident in the 3D rendering of the diffraction pattern in Figure $12[109,110,117]$.

The typical BCDI experimental geometry is shown in Figure 12. The detector can be set to a wide range of diffraction angles. Standard coin cells with a small hole punched in the casing allow the $x$-ray beam to pass through the entire volume of the cell. A detailed description of the instrument configuration and experimental parameters at the Advanced Photon Source Beamline 34-ID-C is available in Li et al. [117] At beamline 34-ID-C of the Advanced Photon Source, the x-ray spot is focused to about $700 \mathrm{~nm}$. Ideally, objects around this size or smaller are imaged. 


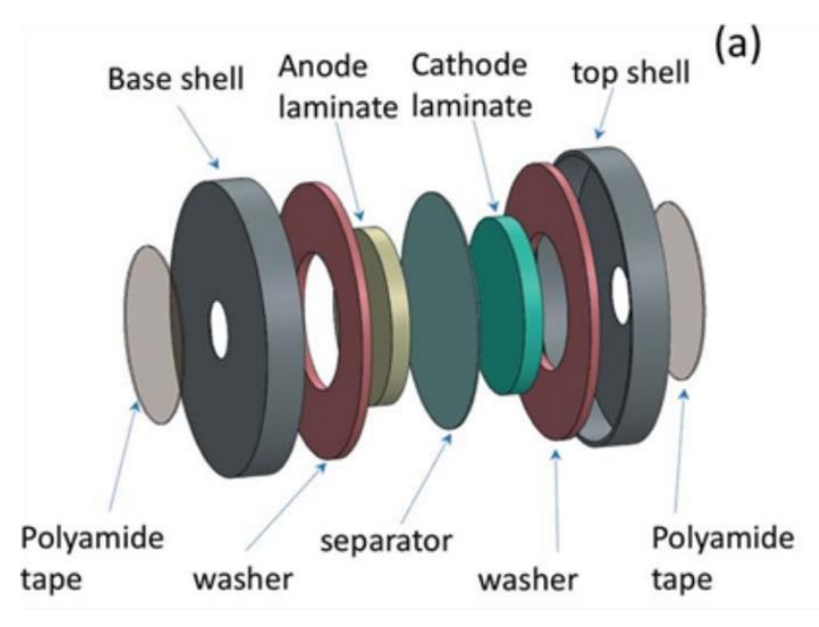

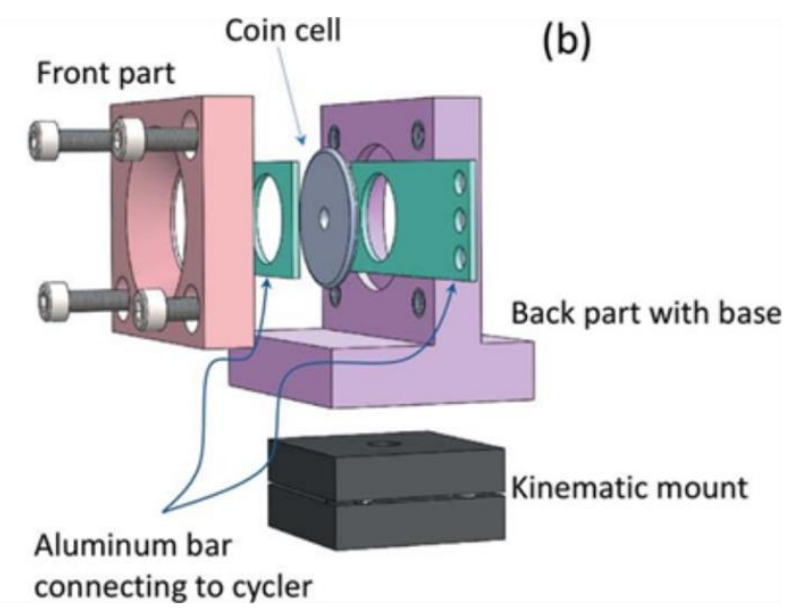

Slice I

Slice II
Slice III (c)
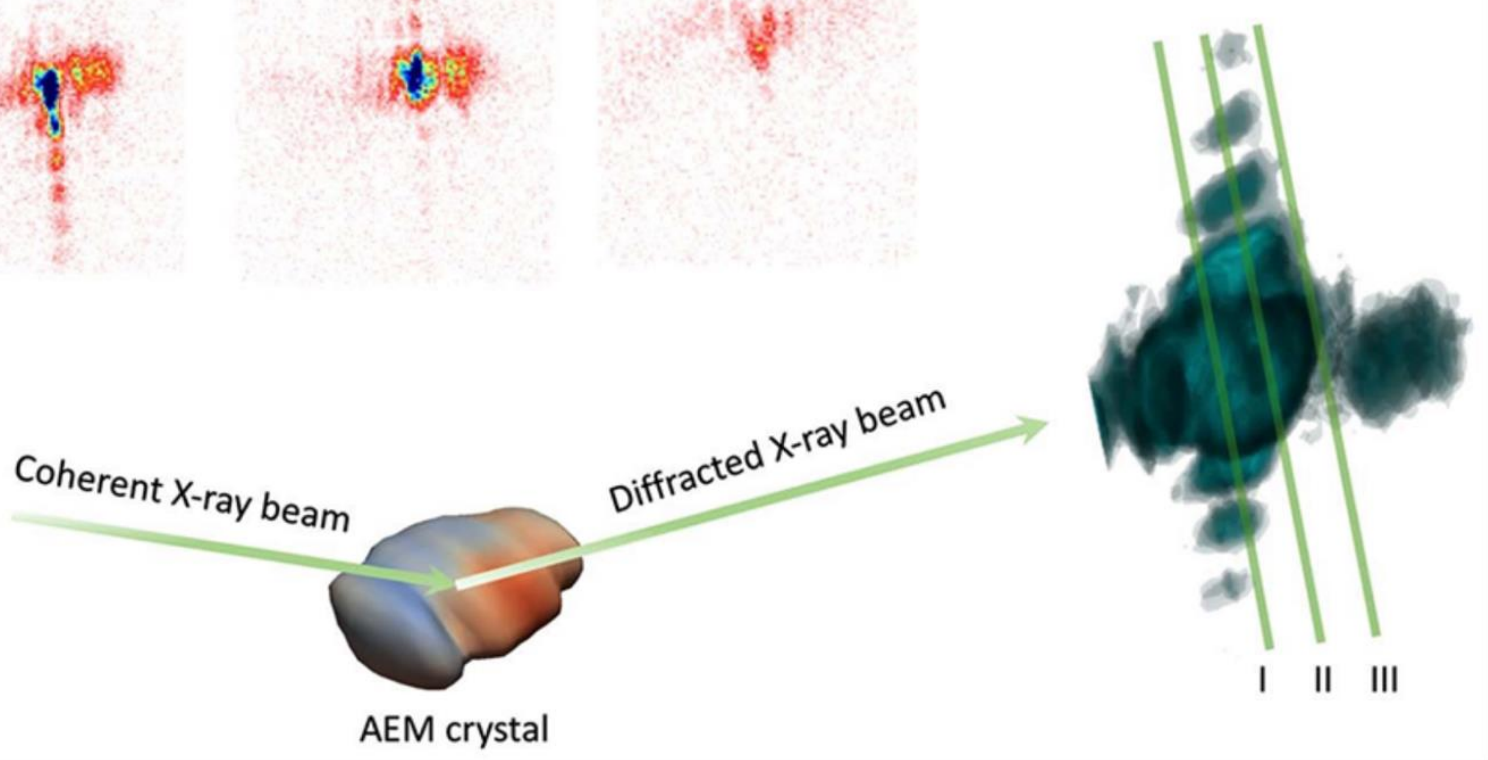

Figure 12. (a) A typical coin cell for in situ battery studies showing the hole in the metal casing allowing the $x$-ray beam to pass through the electrodes. (b) A holder for a coin cell used for operando BCDI of battery materials. (c) The geometry of a BCDI measurement showing the 3D coherent diffraction near a Bragg peak and the raw as seen on the detector as slices through the 3D reciprocal space volume. The volume is measured by rotating the sample through a small angular range. [117]

The first example of operando BCDI was published in 2014 by Ulvestad et al [115]. Here an octahedral spinel nanocrystal $\left(\mathrm{LiNi}_{0.5} \mathrm{Mn}_{1.5} \mathrm{O}_{4}\right)$ was imaged using the (111) Bragg reflection during charge/discharge cycles. Here the change in the angle of the Bragg reflection was used to monitor the average lattice spacing in the particle while the local distortion of the lattice was monitored with BCDI. The evolution of the local lattice distortion is explained by various effects during a $\mathrm{C} / 2$ (dis)charge cycle using the distortion of the lattice as an indirect indication of local lithium concentration. While discharge is initially governed by solid 
solution the particle structure evolves into a striped domain structure governed by coherency strain as shown in Figure 13 (left). The strain is finally released upon a structural phase transformation at $4.2 \mathrm{~V}$, where the lattice again becomes more homogeneous throughout the volume of the crystal. This observation sets a maximum particle size for two-phase coexistence at the width of a domain wall, $50 \mathrm{~nm}$ in the particle observed. Particles below $50 \mathrm{~nm}$ in size should evolve purely through the solid solution. Additionally, they observed hysteresis on the single particle level though analysis of the elastic strain energy held within the particle during (dis)charge, shown in Figure 13 (right). They observed that during charge the elastic energy per unit cell is significantly greater, leading possibly to increased damage of the crystal structure and potential reduction of capacity. The hysteresis is likely caused by energy released in the form of heat, sound, cracks and dislocation formation below the spatial resolution of this imaging experiment.

During a similar experiment, while imaging the behavior of spinel cathode particle during (dis)charge, Ulvestad et al. observed the disconnection of one of the particles they were tracking [114]. The particle was observed to simply stop responding to changes in the potential the cell. Both the position of the Bragg peak remained static, indicating no changes in the average lattice spacing of the crystal were taking place, and the BCDI showed little redistribution of the internal strain structure of the crystal. The authors note that several factors could result in this behavior. The particle could have been electrically disconnected from the electrode through movement or changes in the bulk structure of the cathode, isolation from electrolyte carrying lithium ions, or electrochemically isolated through the formation of a surface layer that impedes the transport of lithium ions into the lattice, such as a solid electrolyte interface layer (SEI). Through close investigation of the strain within the lattice, it was observed that significant residual lithium was concentrated near the surface of the crystal, indicating that and SEI was indeed the cause of this disconnection event.
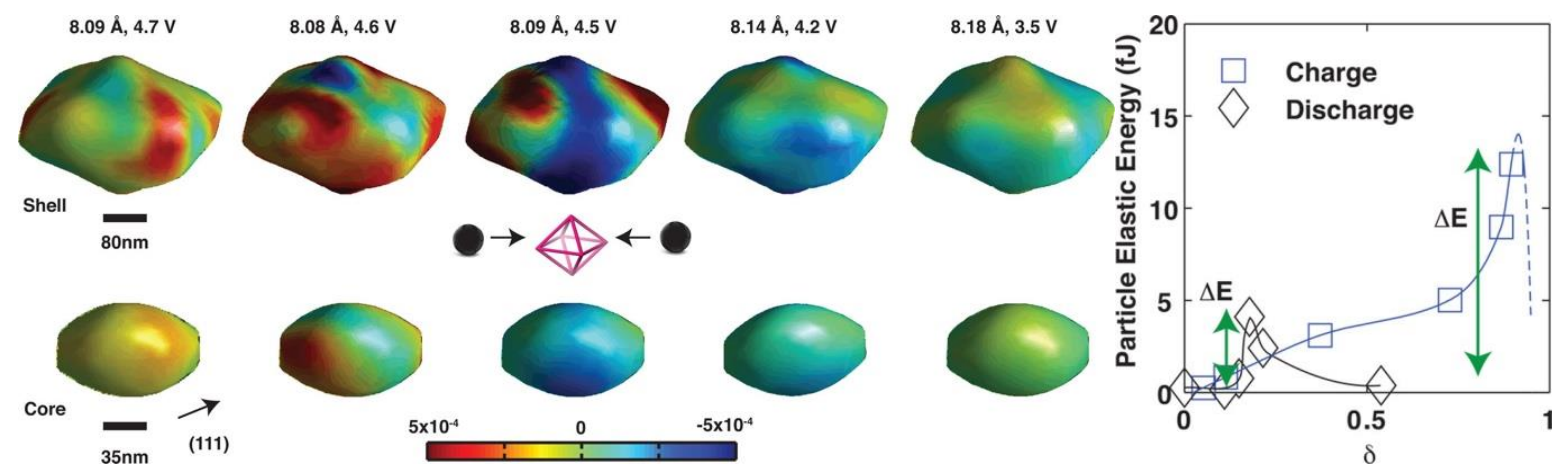

Figure 13. (left) Stripe domains imaged in a single spinel cathode particle during discharge. (right) the elastic strain energy contained in the lattice as a function of lithiation. The large offset between charge and discharge illustrates hysteresis at the single particle level. Reprinted with permission from Ref. [115]. Copyright 2014 American Chemical Society.

In a very recent study using BCDI to investigate the origins of voltage fade in Lithium Rich Layered Oxide (LRLO) materials, Singer et al. found that crystallographic defects are at the heart of this effect [113]. The authors discovered that above $4.2 \mathrm{~V}$ during charge, a network of defects, including screw, edge and partial dislocations formed throughout the particle under study. The paths of the dislocations through the crystal are shown in Figure 14 as lines of spheres. The color of the spheres indicated the type of dislocation being 
traced. The authors claim that while the strain energy is trivial compared to the total energy in the fully charged particle, the dislocations create a severe change in the lithium environment within the layers. The rearrangement stabilizes a lower energy configuration of lithium that persists even when dislocations move out of the lattice or annihilate each other. The authors also show that annealing the cathode material returns it to the high voltage configuration. The LRLO was also compared to identical imaging done on the typical layered oxide $\left(\mathrm{LiNi}_{0.80} \mathrm{CO}_{0.15} \mathrm{Al}_{0.05} \mathrm{O}_{2}\right) \mathrm{NCA}$. While dislocations also appeared in NCA, they observed that the numbers to not increase at a greater charge and actually decrease above $4.2 \mathrm{~V}$.

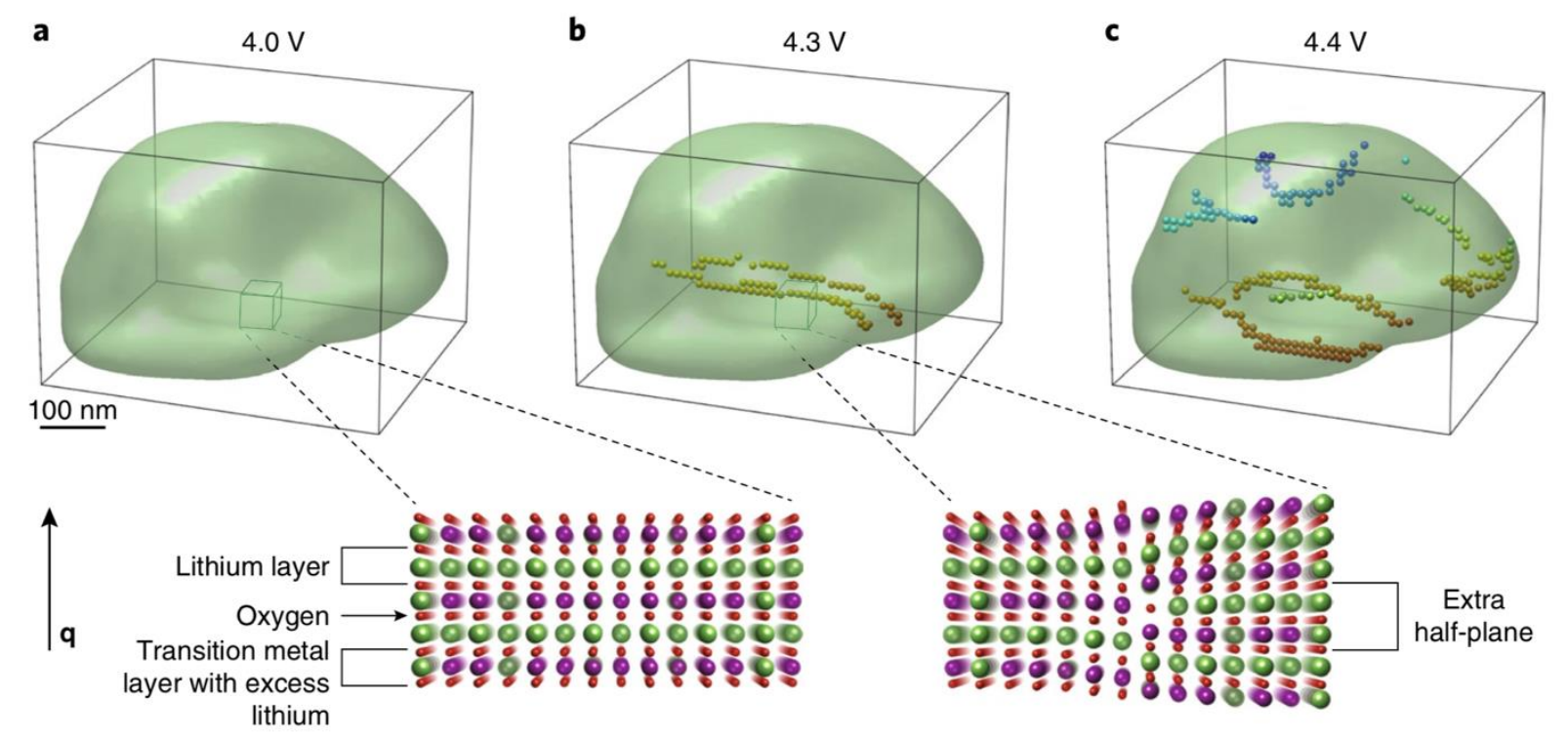

Figure 14. BCDI of LRLO during charge, tracking dislocations moving through the volume of the crystal. Dislocation lines are denoted by the spherical markers whose color indicated the type of defect. Reproduced with permission from Ref. [113]. Copyright 2018, Springer Nature.

Table 1. Summarize the X-ray imaging methods.

\begin{tabular}{|l|l|l|l|l|l|l|}
\hline & $\begin{array}{l}\text { Working } \\
\text { principle }\end{array}$ & Dimensions & Resolution & $\begin{array}{l}\text { Energy } \\
\text { range }\end{array}$ & $\begin{array}{l}\text { Chemical } \\
\text { info }\end{array}$ & Special features \\
\hline $\begin{array}{l}\text { X-ray projection } \\
\text { imaging }\end{array}$ & Transmission & $2 \mathrm{D} / 3 \mathrm{D}$ & $\sim 10 \mu \mathrm{m}$ & $>5 \mathrm{keV}$ & no & Full field imaging \\
\hline TXM & Trasmission & $2 \mathrm{D} / 3 \mathrm{D}$ & $20-30 \mathrm{~nm}$ & $>5 \mathrm{keV}$ & yes & Fast data acquisition \\
\hline STXM & $\begin{array}{l}\text { Transmission } \\
\text { and } \\
\text { fluorescence }\end{array}$ & $2 \mathrm{D}$ & $12-40 \mathrm{~nm}$ & $<2 \mathrm{keV}$ & yes & fight imaging \\
\hline
\end{tabular}




\begin{tabular}{|l|l|l|l|l|l|l|}
\hline & & & & & & $\begin{array}{l}\text { Fluorescence and } \\
\text { electron yield can also } \\
\text { be applied }\end{array}$ \\
\hline $\begin{array}{l}\text { Tender and soft X- } \\
\text { ray imaging }\end{array}$ & Fluorescence & $2 \mathrm{D}$ & $\sim 10 \mu \mathrm{m}$ & $\begin{array}{l}180 \mathrm{ev} \sim \\
5 \mathrm{keV}\end{array}$ & yes & $\begin{array}{l}\text { Covers } \\
\text { traditionally } \\
\text { challenging photon } \\
\text { energy region }\end{array}$ \\
\hline $\mathrm{CDI}$ & Scattering & 2D/3D & Tens of $\mathrm{nms}$ & $>5 \mathrm{keV}$ & no & $\begin{array}{l}\text { Lattice and strain } \\
\text { analysis }\end{array}$ \\
\hline
\end{tabular}

\section{Summary and outlook}

X-ray imaging techniques play a key role in understanding the reaction mechanisms of energy materials. We have reviewed the major $X$-ray imaging techniques, focusing on their working principles and how they have been applied in energy materials research. The main features of the X-ray imaging techniques discussed are summarized in Table 1.

X-ray imaging can provide 2D and 3D morphology information of the enegy materials and devices across a range of length scales from tens of nanometers to tens of micrometers. The chemical mapping capability of the spectromicroscopy techniques, such as the XAS and the XRF mapping in TXM, STXM, and tender and soft X-ray imaing, can probe the elemental distribution and heterogeneities in phase and oxidation states. CDI combines microscopy with diffraction technique, which can map the different domains in a particle as well as the lattice strains and dislocations. Additionally, the sufficient time resolution of X-ray imaging techniques with the non-destructive and high-penetration X-rays makes in-situ/operando characterizations possible. These in-situ/operando experiments have been effectively applied to a range of energy materials to track the structural and chemical changes during device operation in real time, and are essential in understanding the operation mechanisms of energy materials.

Based on the research goals and sample specifications, researchers can choose from the various techniques with different imaging principles and different $X$-ray energies. To be specific, the $X$-ray projection imaging and TXM are full-field imaging techniques which can probe the 2D/3D multi-scale characterization of samples, while STXM builds up the 2D image through raster mapping. Both TXM and STXM can give chemical information when combined with spectroscopy technique. Tender and soft X-ray imaging uses XRF to give both spatial and chemical information and fills the gap in the traditionally more challenging energy region. $\mathrm{CDI}$ is a lensless imaging technique that reconstructs the real space images from scattering in the reciprocal space through computational algorithms, giving crystalline information.

In recent years, large amount of attention has been drawn on applying imaging techniques in energy materials, with studies focused on the macro- and micro-structures of particles, the chemical, morphological, and lattice/strain evolution, and in-situ/operando research. With the progress in the hardware (focusing lens, detectors, etc.) and software (data collecting, storage, and analysis), the X-ray 
imaging techniques will be improved in aspects including but not limited to element sensitivity, spatial and temporal resolution, and reducing beam damage. More advanced techniques continue to emerge, and they will have far-reaching impact on many scientific disciplines. We expect that the development of X-ray imaging methods will aid in the energy materials research and elucidating the complex energy conversion processes, and the rapid growth of these applications will, in turn, accelerate the progress of imaging technologies.

\section{Acknowledgments}

This work was supported by HIT "Young Scientist Studio" and the Start-up Funds from Harbin Institute of Technology, HIT Research Institute (Zhao Yuan) of New Materials and Intelligent Equipment Technology Co., Ltd. Scientific and Technological Cooperation and Development Fund (No.2017KJHZO02).

\section{References}

[1] Li, W., et al., Small Methods (2018) 2 (8), 1700341

[2] Zhou, Y., et al., Joule (2019) 3 (3), 641

[3] Ma, Z., et al., Energy \& Environmental Science (2015) 8 (8), 2144

[4] Zeis, R., Beilstein journal of nanotechnology (2015) 6, 68

[5] Lin, F., et al., Chemical reviews (2017) 117 (21), 13123

[6] Bhattacharyya, R., et al., Nat Mater (2010) 9 (6), 504

[7] Wang, C.-M., Journal of Materials Research (2014) 30 (3), 326

[8] Microscopy and Microanalysis (2018) 24 (S2)

[9] Eastwood, D. S., et al., Chemical communications (2015) 51 (2), 266

[10] Eastwood, D., et al., Nuclear Instruments and Methods in Physics Research Section B: Beam Interactions with Materials and Atoms (2014) 324, 118

[11] Zielke, L., et al., Scientific reports (2015) 5, 10921

[12] Haibel, A., et al., Journal of the Electrochemical Society (2010) 157 (4), A387

[13] Shearing, P. R., et al., Electrochemistry Communications (2010) 12 (3), 374

[14] Shearing, P. R., et al., Journal of The Electrochemical Society (2012) 159 (7), A1023

[15] Gelb, J., et al., Journal of Power Sources (2017) 357, 77

[16] Waldmann, T., et al., Journal of The Electrochemical Society (2016) 163 (10), A2149

[17] Kehrwald, D., et al., Journal of The Electrochemical Society (2011) 158 (12), A1393

[18] Cooper, S., et al., Journal of Power Sources (2014) 247, 1033

[19] Ebner, M., et al., Advanced Energy Materials (2014) 4 (5), 1301278

[20] Zielke, L., et al., Advanced Energy Materials (2014) 4 (8), 1301617

[21] Cooper, S., et al., SoftwareX (2016) 5, 203

[22] Gostick, J., et al., Computing in Science \& Engineering (2016) 18 (4), 60

[23] Chung, D.-W., et al., Journal of The Electrochemical Society (2014) 161 (3), A422

[24] Finegan, D. P., et al., Energy \& Environmental Science (2017) 10 (6), 1377

[25] Finegan, D. P., et al., Nature communications (2015) 6, 6924

[26] Ebner, M., et al., Science (2013) 342 (6159), 716

[27] Taiwo, O. O., et al., Journal of Power Sources (2017) 342, 904

[28] Paz-Garcia, J., et al., Journal of Power Sources (2016) 320, 196

[29] Zielke, L., et al., ChemElectroChem (2016) 3 (7), 1170

[30] Pietsch, P., et al., Scientific reports (2016) 6, 27994

[31] Eastwood, D. S., et al., Advanced Energy Materials (2014) 4 (4), 1300506 
[32] Pietsch, P., et al., Nature communications (2016) 7, 12909

[33] Tan, C., et al., ACS Applied Energy Materials (2018) 1 (9), 5090

[34] Yufit, V., et al., Joule (2019) 3 (2), 485

[35] Robinson, J. B., et al., Journal of Power Sources (2018) 400, 360

[36] Gibbs, J., et al., Scientific reports (2015) 5, 11824

[37] Harry, K. J., et al., Nature materials (2014) 13 (1), 69

[38] Shen, F., et al., ACS Energy Letters (2018) 3 (4), 1056

[39] Dixit, M. B., et al., ACS applied materials \& interfaces (2019) 11 (2), 2022

[40] Sinha, P. K., et al., Electrochemical and Solid-State Letters (2006) 9 (7), A344

[41] Becker, J., et al., Journal of fuel cell science and technology (2008) 5 (2), 021006

[42] Gostick, J. T., et al., Journal of Power Sources (2007) 173 (1), 277

[43] Bazylak, A., International journal of hydrogen energy (2009) 34 (9), 3845

[44] Zenyuk, I. V., Current Opinion in Electrochemistry (2018)

[45] Meyer, Q., et al., Electrochimica Acta (2016) 211, 478

[46] Zenyuk, I. V., et al., Journal of Power Sources (2016) 328, 364

[47] Eberhardt, S., et al., Journal of The Electrochemical Society (2016) 163 (8), F842

[48] Eller, J., et al., ECS Transactions (2015) 69 (17), 523

[49] Shum, A. D., et al., Electrochimica Acta (2017) 256, 279

[50] Hack, J., et al., Journal of The Electrochemical Society (2018) 165 (6), F3045

[51] Hoeh, M. A., et al., Electrochemistry communications (2015) 55, 55

[52] Leonard, E., et al., Electrochimica Acta (2018) 276, 424

[53] Suermann, M., et al., Journal of The Electrochemical Society (2017) 164 (9), F973

[54] Wang, J., et al., Chemical communications (2013) 49 (58), 6480

[55] Wang, L., et al., Small Methods (2018) 2 (8), 1700293

[56] Nelson Weker, J., and Toney, M. F., Advanced Functional Materials (2015) 25 (11), 1622

[57] Wang, J., et al., Nature communications (2014) 5, 4570

[58] Chen-Wiegart, Y.-c. K., et al., (2013) 8851, 88510C

[59] Meirer, F., et al., Journal of synchrotron radiation (2011) 18 (Pt 5), 773

[60] Yao, S., et al., NNLSF: A fast and informative fitting method for XANES chemical mapping analysis. In

2015 IEEE 12th International Symposium on Biomedical Imaging (ISBI), IEEE(2015), pp 1155

[61] Liu, Y., et al., Journal of synchrotron radiation (2012) 19 (Pt 2), 281

[62] Nelson, J., et al., Journal of the American Chemical Society (2012) 134 (14), 6337

[63] Grew, K. N., et al., Journal of The Electrochemical Society (2010) 157 (6), B783

[64] Ruther, R. E., et al., Chemistry of Materials (2017) 29 (7), 2997

[65] Tian, C., et al., Joule (2018) 2 (3), 464

[66] Wang, J., et al., Nature communications (2015) 6, 7496

[67] Wang, J., et al., Angewandte Chemie (2014) 53 (17), 4460

[68] Kan, W. H., et al., Advanced Functional Materials (2019), 1808294

[69] Wang, J., et al., Nature communications (2016) 7, 12372

[70] Behrens, S., et al., Environmental microbiology (2012) 14 (11), 2851

[71] Liu, J., et al., Canadian Journal of Chemistry (2017) 95 (11), 1163

[72] Lim, J., et al., Science (2016) 353 (6299), 566

[73] de Smit, E., et al., Nature (2008) 456 (7219), 222

[74] Luhl, L., et al., Journal of synchrotron radiation (2019) 26 (Pt 2), 430

[75] Zhou, J., et al., Physical chemistry chemical physics : PCCP (2016) 18 (33), 22789

[76] Behyan, S., et al., Surface detection in a STXM microscope. In AIP Conference Proceedings, AIP(2011), Vol. 1365, pp 184

[77] Hitchcock, A. P., Journal of Electron Spectroscopy and Related Phenomena (2015) 200, 49 
[78] Advanced Photon Source microscopy beamlines. www.aps.anl.gov/Microscopy/Beamlines, (2018)

[79] Vantelon, D., et al., Journal of synchrotron radiation (2016) 23 (2), 635

[80] PHOENIX X07MA/B. www.psi.ch/de/sls/phoenix, (2019)

[81] Hu, Y., et al., Preliminary commissioning and performance of the soft $\mathrm{X}$ - ray micro -

characterization beamline at the Canadian Light Source. In AIP Conference Proceedings, AIP(2010), Vol. 1234, pp 343

[82] Xiao, Q., et al., Journal of synchrotron radiation (2017) 24 (1), 333

[83] Xiao, Q., et al., Microscopy and Microanalysis (2018) 24 (S2), 466

[84] Regier, T., et al., Nuclear Instruments and Methods in Physics Research Section A: Accelerators, Spectrometers, Detectors and Associated Equipment (2007) 582 (1), 93

[85] Wang, D., et al., Journal of Power Sources (2016) 302, 223

[86] Wang, D., et al., The Journal of Physical Chemistry C (2015) 119 (34), 19573

[87] Turnbull, M. J., et al., The Journal of chemical physics (2016) 145 (20), 204702

[88] Banis, M. N., et al., The Canadian Journal of Chemical Engineering

[89] Nishijima, M., et al., Nature communications (2014) 5, 4553

[90] Xu, Z., et al., Journal of The Electrochemical Society (2016) 163 (13), A2600

[91] Amin, R., et al., Advanced Functional Materials (2009) 19 (11), 1697

[92] Neuville, D. R., et al., Chemical Geology (2004) 213 (1-3), 153

[93] Banis, M. N., et al., Energy \& Environmental Science (2018) 11 (8), 2073

[94] Landa-Medrano, I., et al., Nano energy (2017) 37, 224

[95] Hu, Y., et al., VLS - PGM Beamline at the Canadian Light Source. In AIP conference proceedings, $\operatorname{AIP}(2007)$, Vol. 879, pp 535

[96] Sham, T. K., Synchrotron Radiation in Materials Science: Light Sources, Techniques, and

Applications. John Wiley \& Sons(2018), Vol. 1, pp 123

[97] Heigl, F., et al., Communication: X-ray excited optical luminescence from $\mathrm{TbCl} 3$ at the giant resonance of terbium. AIP(2013)

[98] Sham, T. K., and Gordon, R. A., RIXS, XEOL and XEOL Imaging of Rare - earth Phosphors at the L 3, 2 - edges. In AIP Conference Proceedings, AIP(2010), Vol. 1234, pp 133

[99] Wang, Z., et al., Microscopy and Microanalysis (2018) 24 (S2), 480

[100] Miao, J., et al., Nature (1999) 400 (6742), 342

[101] Fienup, J. R., Applied optics (1982) 21 (15), 2758

[102] Clark, J. N., et al., Nature materials (2015) 14 (8), 780

[103] Clark, J., et al., Nature communications (2012) 3, 993

[104] Takahashi, Y., et al., Physical Review B (2009) 80 (5), 054103

[105] Huang, X., et al., Scientific reports (2013) 3, 3562

[106] Diaz, A., et al., Physical Review B (2012) 85 (2), 020104

[107] Shapiro, D. A., et al., Nature Photonics (2014) 8 (10), 765

[108] Bragg, W. H., and Bragg, W. L., Proceedings of the Royal Society of London. Series A, Containing

Papers of a Mathematical and Physical Character (1913) 88 (605), 428

[109] Pfeifer, M. A., et al., Nature (2006) 442 (7098), 63

[110] Robinson, I., and Harder, R., Nature materials (2009) 8 (4), 291

[111] Rodenburg, J., et al., Physical review letters (2007) 98 (3), 034801

[112] Singer, A., et al., Nano letters (2014) 14 (9), 5295

[113] Singer, A., et al., Nat. Energy (2018) 3 (8), 641

[114] Ulvestad, A., et al., Physical Chemistry Chemical Physics (2015) 17 (16), 10551

[115] Ulvestad, A., et al., Nano letters (2014) 14 (9), 5123

[116] Ulvestad, A., et al., Science (2015) 348 (6241), 1344

[117] Li, L., et al., Journal of synchrotron radiation (2019) 26 (1) 
